\title{
Isotropic Polyharmonic B-Splines: Scaling Functions and Wavelets
}

\author{
Dimitri Van De Ville, Member, Thierry Blu, Member, and Michael Unser, Fellow, IEEE
}

\begin{abstract}
In this paper, we use polyharmonic B-splines to build multidimensional wavelet bases. These functions are nonseparable, multidimensional basis functions that are localized versions of radial basis functions. We show that Rabut's elementary polyharmonic B-splines do not converge to a Gaussian as the order parameter increases, as opposed to their separable B-spline counterparts. Therefore, we introduce a more isotropic localization operator that guarantees this convergence, resulting into the isotropic polyharmonic B-splines. Next, we focus on the two-dimensional quincunx subsampling scheme. This configuration is of particular interest for image processing because it yields a finer scale progression than the standard dyadic approach. However, up until now, the design of appropriate filters for the quincunx scheme has mainly been done using the McClellan transform. In our approach, we start from the scaling functions, which are the polyharmonic B-splines and, as such, explicitly known, and we derive a family of polyharmonic spline wavelets corresponding to different flavors of the semi-orthogonal wavelet transform; e.g., orthonormal, B-spline, and dual. The filters are automatically specified by the scaling relations satisfied by these functions. We prove that the isotropic polyharmonic B-spline wavelet converges to a combination of four Gabor atoms, which are well separated in the frequency domain. We also show that these wavelets are nearly isotropic and that they behave as an iterated Laplacian operator at low frequencies. We describe an efficient fast Fourier transform-based implementation of the discrete wavelet transform based on polyharmonic B-splines.
\end{abstract}

Index Terms-Gabor wavelets, isotropy, multiresolution analysis, polyharmonic B-splines, quincunx lattice, rotation invariance, scaling functions, wavelets.

\section{INTRODUCTION}

$\mathbf{M}$ ULTIRESOLUTION analysis has proved to be a powerful framework for providing time-frequency localized expansions with applications in many areas of signal processing. The well-known one-dimensional (1-D) algorithm for the discrete wavelet transform corresponds to a two-channel filterbank: It splits the signal into two subsampled channels (at the analysis side), and it offers perfect reconstruction after upsampling and filtering again at the synthesis side. The wavelet transform can also be formulated in the continuous time domain, an approach that is often favored by mathematicians [1], [2]. Instead of working directly with the filters, this representation considers continuously defined basis functions, i.e., scaling functions and

Manuscript received February 20, 2004; revised July 30, 2004. This work was supported in part by the Swiss National Science Foundation under Grants 200020-101821 and 200020-109415. The associate editor coordinating the review of this manuscript and approving it for publication was Dr. Ivan W. Selesnick.

The authors are with the Biomedical Imaging Group, Swiss Federal Institute of Technology Lausanne (EPFL), CH1015 Lausanne, Switzerland (e-mail: dimitri.vandeville@epfl.ch; thierry.blu@epfl.ch; michael.unser@epfl.ch).

Digital Object Identifier 10.1109/TIP.2005.857249 wavelets. Recently, it has been demonstrated that the scaling function can always be expressed as a convolution of a B-spline (its regular part) and a residual distribution without order nor smoothness [3].

In the context of image processing, the most frequently used two-dimensional (2-D) wavelet transforms are separable; their basis functions and filters are simply tensor products of the 1-D ones. Unfortunately, these decompositions introduce preferred (vertical and horizontal) directions and create a "diagonal" cross term that does not have a straightforward interpretation. This has motivated researchers to design better nonseparable wavelet transforms. One option is to privilege angular selectivity. Numerous directional wavelet transforms, both frames (i.e., redundant) and bases (i.e., nonredundant), have been proposed [4]-[11]. Such representations can serve to sparsely represent essential image features such as edges combined with their orientation. Another interesting option, which has received less attention, is to emphasize isotropy. A strong motivation for this kind of design is that many standard image processing algorithms exploit the rotation-invariant properties of filters such as the Gaussian and Laplacian.

Our construction starts from radial basis functions (RBFs), which are isotropic versions of power functions. Rabut has introduced a scheme to produce basis functions that are localized versions of these RBFs - the so-called "elementary polyharmonic B-splines.” These B-splines share many interesting properties with their classical 1-D counterparts. While studying these functions we discovered that, contrary to our expectations, they fail to converge to a Gaussian as the order increases. Since it is very desirable to have Gaussian-like basis functions, which are isotropic and optimally localized in space frequency in the sense specified by the uncertainty principle, we decided to further investigate this issue. This led us to the construction of the "isotropic polyharmonic B-splines," which is presented in Section II. Our scheme utilizes a more isotropic discretization of the Laplacian operator, which guarantees the desired Gaussian convergence property. The generalization remains valid in higher dimensions as well.

The isotropic polyharmonic B-spline is an interesting candidate to be used as a scaling function for a wavelet decomposition. First, it has been shown that any multidimensional scaling function of order $\gamma$ can be represented as the convolution of a polyharmonic B-spline of order $\gamma$ and a distribution with a bounded Fourier transform [12], [13]. It is the polyharmonic B-spline that brings all the desirable mathematical properties. Second, the isotropic polyharmonic B-spline satisfies a wide variety of scaling relations; in fact, many more than the classical dyadic ones. Here, we will focus on a particularly interesting 
one in two dimensions: the quincunx subsampling scheme. This scheme provides a more progressive transition through scale than the dyadic subsampling scheme [14]-[19]. For each iteration, the number of samples is halved, so a single wavelet can be used to characterize each bandpass subband. Up to now, most research for quincunx subsampling has focused on filterbank design and it has been difficult to define general wavelet families, as in one dimension, due to the lack of multivariate factorization theorems. One popular option to circumvent this difficulty is to use the McClellan transform that maps a 1-D design into a 2-D one [20]-[22].

In Section III, we consider an alternative approach and introduce new wavelet bases for the quincunx subsampling scheme based on the 2-D isotropic polyharmonic B-splines. Specifically, we present a semi-orthogonal design procedure that yields a complete family of polynomial isotropic polyharmonic B-spline wavelets. We demonstrate that these wavelets form Riesz bases and that they qualitatively behave as a $\gamma$ th-order Laplacian operator for low frequencies, which is isotropic once more. We also prove that one particular wavelet-the isotropic polyharmonic B-spline wavelet-converges to the sum of four Gabor atoms as the order increases. Additionally, it behaves as (fractionally) iterated Laplacian (and, therefore, isotropic) operator for low frequencies. Clearly, in our design, the continuously defined functions (scaling functions and wavelets) play a central role. Nevertheless, the associated filters, which are required to implement the transform, are automatically defined. Explicit formulas are given in the Fourier domain. The implementation of the discrete wavelet transforms is presented in Section IV.

\section{POLYHARMONIC B-SPlines}

\section{A. Univariate Case: B-Spline Basis Functions and Signal Spaces}

The construction of the polyharmonic B-splines is best explained by using the analogy with the standard 1-D B-splines. In particular, we consider the symmetric B-spline of odd degree $2 m-1, m \in \mathbb{N} \backslash\{0\}$. Its Fourier transform is given by

$$
\hat{\beta}^{2 m-1}(\omega)=\left(\frac{4 \sin ^{2}(\omega / 2)}{\omega^{2}}\right)^{m}
$$

We recognize the localization filter as the numerator and the power function as the denominator. In the spatial domain, such a B-spline can be regarded as a localized version of the two-sided power function $|x|^{2 m-1}$ [23]. Consequently, the B-splines are piecewise polynomials.

The B-splines span the integer-shift-invariant signal space

$$
\mathcal{V}\left(\beta^{2 m-1}\right)=\left\{s(x)=\sum_{k} c(k) \beta^{2 m-1}(x-k), c \in l_{2}(\mathbb{Z})\right\}
$$

where $c(k)$ are coefficients that are used as weights for the shifted basis functions. The notation $\sum_{k}$ stands for the sum of all $k \in \mathbb{Z}$. An essential property [24] of each spline signal $s(x)$ of $\mathcal{V}\left(\beta^{2 m-1}\right)$ is that they satisfy

$$
\frac{d^{2 m} s(x)}{d x^{2 m}}=0, \quad \text { for } x \in \mathbb{R} \backslash \mathbb{Z} .
$$

In fact, the two-sided power function $|x|^{2 m-1}$ is the symmetric Green function (or fundamental solution) of the differential operator in (3), i.e., the solution of the differential operator that gives $\delta(x)$. This implies, among other things, that the signal space $\mathcal{V}\left(\beta^{2 m-1}\right)$ is also spanned by shifted versions of $|x|^{2 m-1}$ [23]. Another important observation is that the localization filter of the B-spline, represented by the numerator of (1), is the most elementary discretization of the corresponding "ideal" differential operator ( $m$ th iterate of a second derivative).

\section{B. Polyharmonic Case: From RBFs to B-Splines}

Historically, the first extension of the univariate spline principles to $N$ dimensions-next to tensor products-consists of building splines $s(\mathbf{x}), \mathbf{x} \in \mathbb{R}^{N}$, that satisfy

$$
\Delta^{m} s(\mathbf{x})=0, \quad \text { for } \mathbf{x} \in \mathbb{R}^{N} \backslash \mathbb{Z}^{N}
$$

where $\Delta=\sum_{i=1}^{N} \partial^{2} / \partial x_{i}^{2}$ denotes the Laplacian operator. As in the 1-D case, such splines $s(\mathbf{x})$ can be represented as linear combinations of shifted Green functions of the $m$-iterated Laplacian, which are known to be

$$
\begin{aligned}
\rho(\mathbf{x}) & =\|\mathbf{x}\|^{2 m-N}\left(c_{N, m} \ln \|\mathbf{x}\|+c_{N, m}^{\prime}\right) \\
\longleftrightarrow \quad \hat{\rho}(\boldsymbol{\omega}) & =\frac{1}{\|\boldsymbol{\omega}\|^{2 m}}
\end{aligned}
$$

where $c_{N, m}$ and $c_{N, m}^{\prime}$ are some suitable constants ( $c_{N, \gamma}^{\prime}$ is zero when $2 m-N$ is even, while $c_{N, \gamma}$ is zero otherwise). The functions $\rho(\mathbf{x})$ are better known as generalized thin plate splines, an interesting class of RBFs) Each spline in the space spanned by this type of RBFs can be written as

$$
s(\mathbf{x})=\sum_{\mathbf{k}} c(\mathbf{k}) \rho(\mathbf{x}-\mathbf{k})
$$

and will necessarily satisfy (4). Often a polynomial of degree $m-1$ is added to the right-hand side of (6), however, on the (infinite) Cartesian grid $\mathbb{Z}^{N}$, this polynomial is automatically included in the closure of the span of the RBFs. These splines have been studied intensively for (finite) scattered data interpolation [25]-[31] and also for the representation of signals on a uniform grid [32]-[34]. Probably, the earliest example has been given by Harder [25], which corresponds to $N=2$ and $m=2$.

The direct application of RBFs to interpolation, as in (6), poses various theoretical and practical difficulties. Therefore, it is interesting to look for better conditioned basis functions, such as B-splines, that span the same signal space but are essentially localized. Rabut [35] defined "elementary $m$-harmonic cardinal B-splines" by choosing the localization filter as the most elementary discretization of the Laplacian.

${ }^{1}$ Rabut introduced this terminology: "elementary" to emphasize that they are obtained by using the most elementary discretization of the Laplacian operator; " $m$-harmonic" since they are in the span of the fundamental solution of $\Delta^{m} f=$ $\delta$ (see, also, [32] and [33]); "cardinal" due to the uniform grid; and "B-splines" since they are "bell-shaped" and regularize the Dirac distribution. 


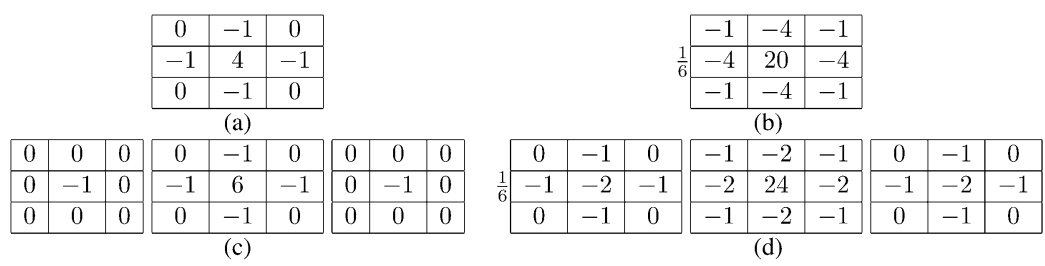

Fig. 1. (a) Filter coefficients for Rabut's 2-D discretization of the Laplacian operator ( $m=1, \gamma=2)$. (b) Filter coefficients for the isotropic 2-D discretization of the Laplacian operator. (c) Filter coefficients for Rabut's 3-D discretization of the Laplacian operator. (d) Filter coefficients for the isotropic 3-D discretization of the Laplacian operator.

Definition 1 (Elementary m-Harmonic Cardinal B-Splines): Their definition in the Fourier domain is remarkably similar to the univariate case

$$
\begin{aligned}
\hat{\phi}^{m}(\boldsymbol{\omega}) & =\frac{\|2 \sin (\boldsymbol{\omega} / 2)\|^{2 m}}{\|\boldsymbol{\omega}\|^{2 m}} \\
& =\left(\frac{4 \sum_{k=1}^{N} \sin ^{2}\left(\omega_{k} / 2\right)}{\sum_{k=1}^{N} \omega_{k}^{2}}\right)^{m}
\end{aligned}
$$

with $\sin (\boldsymbol{\omega})=\left(\sin \left(\omega_{1}\right), \ldots, \sin \left(\omega_{N}\right)\right)$. The parameter $m$ is an integer with $m \geq N / 2$.

In Fig. 1(a) and (c), we show the discretized Laplacian operator $(m=1)$ for the 2-D and three-dimensional (3-D) case. From now on, we prefer to denote the polyharmonic B-splines by their order of approximation, which is given by $\gamma=2 m$ (see later). Therefore, we denote them also as $\phi^{m}=\phi_{2 m}=\phi_{\gamma}$. The signal space generated by $\phi_{\gamma}$, which is the same as that spanned by RBFs of degree $\gamma-N$, can be written as

$$
\mathcal{V}\left(\phi_{\gamma}\right)=\left\{\sum_{\mathbf{k}} c(\mathbf{k}) \phi_{\gamma}(\mathbf{x}-\mathbf{k}), c \in l_{2}\left(\mathbb{Z}^{N}\right)\right\} .
$$

The polyharmonic B-splines defined in this way satisfy most of the properties of the conventional B-splines: close resemblance of their definition in the Fourier domain, convolution relation $\phi_{\gamma_{1}+\gamma_{2}}=\phi_{\gamma_{1}} * \phi_{\gamma_{2}}$, partition of unity, total positivity $\left(\hat{\phi}_{\gamma}>0\right)$. These splines are also reported to be "bell-shaped" functions. Unfortunately, and despite the fact that they are generated by multiple convolutions, they do not converge toward a Gaussian as the order increases.

Proposition 1: The elementary $m$-harmonic cardinal B-splines violate the conditions for the applicability of the central limit theorem and do not converge toward a Gaussian as the order increases.

Proof: The central limit theorem guarantees the convergence of iterated convolutions to a Gaussian. However, it requires a well-defined second-order moment. The second-order moment can be identified in the Fourier domain by considering the Taylor series development of $\hat{\phi}_{2}(\boldsymbol{\omega})$ for $\boldsymbol{\omega} \rightarrow \mathbf{0}$

$$
\begin{aligned}
\hat{\phi}_{2}(\boldsymbol{\omega}) & =\frac{4 \sum_{k=1}^{N} \sin ^{2}\left(\omega_{k} / 2\right)}{\sum_{k=1}^{N} \omega_{k}^{2}} \\
& =1-\frac{1}{12}\left(\frac{\sum_{k=1}^{N} \omega_{k}^{4}}{\sum_{k=1}^{N} \omega_{k}^{2}}\right)+\mathcal{O}\left(\|\boldsymbol{\omega}\|^{4}\right), \quad \boldsymbol{\omega} \rightarrow \mathbf{0}
\end{aligned}
$$

The second term is not twice continuously differentiable at $\mathbf{0}$, which implies that the second-order moment does not exist. ${ }^{2}$ Following the outline of the proof of the central limit theorem, we can find the limiting function, using (9), as

$$
\begin{aligned}
\hat{\phi}_{\gamma}\left(\frac{\omega}{\sqrt{\gamma}}\right) & \approx\left(1-\frac{1}{\gamma} \frac{1}{12}\left(\frac{\sum_{k=1}^{N} \omega_{k}^{4}}{\sum_{k=1}^{N} \omega_{k}^{2}}\right)\right)^{\gamma / 2} \\
& \longrightarrow \exp \left(-\frac{1}{24} \frac{\sum_{k=1}^{N} \omega_{k}^{4}}{\sum_{k=1}^{N} \omega_{k}^{2}}\right), \quad \text { as } \gamma \rightarrow \infty
\end{aligned}
$$

which, clearly, is not a Gaussian.

Rabut [36] also defined " $n$th level $m$-harmonic cardinal B-splines" that were primarily designed to be improved quasi-interpolants, i.e., functions that interpolate polynomials of higher degree than the elementary versions. The higher level polyharmonic splines only converge toward a degenerated Gaussian, namely a Dirac distribution.

\section{Isotropic Polyharmonic B-Splines}

The discretization of the Laplacian operator corresponding to the numerator of (7) uses the least possible number of filter coefficients. The downside of this approach is the nonconvergence to a Gaussian, which can be explained by a lack of isotropy of the discrete approximation of the Laplacian. This motivates us to introduce the "isotropic polyharmonic B-splines" using a slightly different, but more isotropic, discretization of the Laplacian operator. Strictly speaking, these B-splines are only quasi-isotropic, but they do become more and more isotropic as the order increases. Notationally, we usually specify a filter $h(\mathbf{k}), \mathbf{k} \in \mathbb{Z}^{N}$, by its $\mathcal{Z}$-transform as

$$
H(\mathbf{z})=\sum_{\mathbf{k}} h(\mathbf{k}) \mathbf{z}^{-\mathbf{k}}
$$

where $\mathbf{z}^{\mathbf{k}}$ is a shortcut notation for $\prod_{i=1}^{N} z_{i}^{k_{i}}$. We obtain its frequency response by putting $\mathbf{z}=e^{j \boldsymbol{\omega}}$, i.e., $H\left(e^{j \boldsymbol{\omega}}\right)$.

Definition 2 (Isotropic Polyharmonic B-Splines): For $N=$ 2 and 3, the isotropic polyharmonic B-splines of order $\gamma$ are defined in the Fourier domain as

$$
\hat{\beta}_{\gamma}(\boldsymbol{\omega})=\frac{V_{\gamma}\left(e^{j \boldsymbol{\omega}}\right)}{\|\boldsymbol{\omega}\|^{\gamma}}
$$

\footnotetext{
${ }^{2}$ For the 2-D case, it is instructive to switch to polar coordinates, which
} amounts to replacing $\left(\omega_{1}, \omega_{2}\right)$ by $\left(r_{\omega} \cos \left(\theta_{\omega}\right), r_{\omega} \sin \left(\theta_{\omega}\right)\right)$ in (9). 


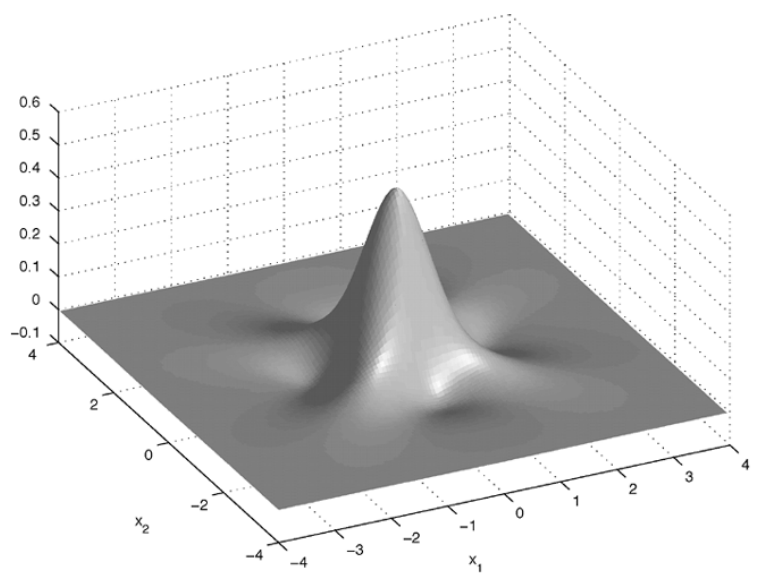

(a)

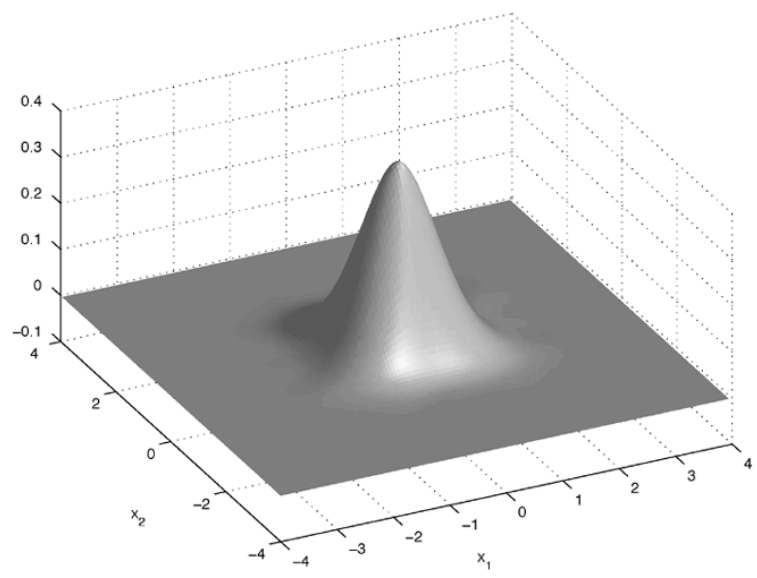

(b)

Fig. 2. Two-dimensional example of polyharmonic B-splines of fifth-order $(N=2, \gamma=5)$. (a) The elementary polyharmonic B-spline. (b) The isotropic polyharmonic B-spline.

where $V_{\gamma}\left(e^{j \boldsymbol{\omega}}\right)=V_{2}\left(e^{j \boldsymbol{\omega}}\right)^{\gamma / 2}$ and

$$
\begin{aligned}
V_{2}\left(e^{j \boldsymbol{\omega}}\right)=4 \sum_{k=1}^{N} & \sin ^{2}\left(\frac{\omega_{k}}{2}\right) \\
& -\frac{8}{3} \sum_{k=1}^{N-1} \sum_{l=k+1}^{N} \sin ^{2}\left(\frac{\omega_{k}}{2}\right) \sin ^{2}\left(\frac{\omega_{l}}{2}\right) .
\end{aligned}
$$

The second term in $V_{2}\left(e^{j \omega}\right)$ is a slight change of the elementary localization operator, but is essential to ensure the Gaussian convergence. Moreover, using standard trigonometric formulæ, one checks that $V_{2}\left(e^{j \omega}\right)$ is positive for $N=2,3$, because it can be expressed as a sum of positive quantities

$$
\begin{aligned}
V_{2}\left(e^{j \boldsymbol{\omega}}\right) & =\frac{4}{3}(4-N) \sum_{k=1}^{N} \sin ^{2}\left(\frac{\omega_{k}}{2}\right) \\
+ & \frac{2}{3} \sum_{k=1}^{N-1} \sum_{l=k+1}^{N}\left(\sin ^{2}\left(\frac{\omega_{k}+\omega_{l}}{2}\right)+\sin ^{2}\left(\frac{\omega_{k}-\omega_{l}}{2}\right)\right) .
\end{aligned}
$$

For $N \geq 4$, it would be necessary to include higher order terms for the localization filter to be strictly positive-when $N=4$, the present filter vanishes at $\omega=(\pi, \pi, \pi, \pi)$ which makes the lower Riesz bound ill defined (see Section II-D2).

Proposition 2: The isotropic polyharmonic B-splines converge to a Gaussian as the order $\gamma$ increases.

Proof: Reconsider the development of the elementary polyharmonic B-splines of (9). Clearly, for the second term to be isotropic, its numerator should be proportional to $\left(\sum_{k=1}^{N} \omega_{k}^{2}\right)^{2}$. This can be obtained by adding the missing crossterms to $\sum_{k=1}^{N} \omega_{k}^{4}$. It can be verified that the new numerator proposed in (10) results into

$$
\hat{\beta}_{\gamma}\left(\frac{\boldsymbol{\omega}}{\sqrt{\gamma}}\right)=1-\frac{1}{24}\|\boldsymbol{\omega}\|^{2}+\mathcal{O}\left(\|\boldsymbol{\omega}\|^{4}\right), \quad \text { as } \boldsymbol{\omega} \rightarrow \mathbf{0}
$$

The second-order moment is now a constant $(\gamma / 12)$, irrespective of the direction. This property ensures that the isotropic polyharmonic B-splines rapidly converge to a Gaussian as the order $\gamma$ increases.

We want to show the effect of the isotropic discretization of the Laplacian (i.e., for $\gamma=2$ ), as compared to the elementary discretization. In the 2-D case, the adjustment term of the localization filter introduces new knots at the corners of the $3 \times 3$, see Fig. 1(b). Interestingly, the proposed isotropic discretization of the Laplacian can also be seen ${ }^{3}$ as the particular combination

$$
V_{2}(\mathbf{z})=\frac{2}{3} L^{+}(\mathbf{z})+\frac{1}{3} L^{\times}(\mathbf{z})
$$

where $L^{+}$represents the elementary discretization along the axes [i.e., the numerator of (7) for $m=1$ ]

$$
L^{+}(\mathbf{z})=4-z_{1}-z_{1}^{-1}-z_{2}-z_{2}^{-1}
$$

and $L^{\times}$the elementary discretization along the diagonals

$$
L^{\times}(\mathbf{z})=\frac{1}{2}\left(4-z_{1} z_{2}-z_{1}^{-1} z_{2}-z_{1} z_{2}^{-1}-z_{1}^{-1} z_{2}^{-1}\right) .
$$

For the 3 -D case, we notice that not all coefficients of the $3 \times 3 \times 3$ cube are required, see Fig. 1(d).

From now on, we consider the definition of the isotropic polyharmonic B-splines for any fractional-order $\gamma \in \mathbb{R}$ with $\gamma>$ $N / 2$, since this extension does not present any theoretical difficulty, as long as we work in the Fourier domain. In Fig. 2, we show the 2-D elementary and isotropic polyharmonic B-spline for $\gamma=5$, respectively. These functions are easy to evaluate numerically, for any order $\gamma$, by resampling (10) with a sufficient number of points and applying an inverse fast Fourier transform (FFT).

\section{Key Properties}

We now present the key properties of the polyharmonic B-splines (both the elementary and the isotropic ones). When necessary, we make a distinction between both.

1) Partition of Unity: The partition of unity property guarantees that the polyharmonic B-splines reproduce the constant

$$
\sum_{\mathbf{n} \in \mathbb{Z}^{N}} \beta_{\gamma}(\mathbf{x}-\mathbf{n})=1 \longleftrightarrow \hat{\beta}_{\gamma}(2 \pi \mathbf{k})=\delta_{\mathbf{k}}, \quad \mathbf{k} \in \mathbb{Z}^{N} .
$$

This can be established directly by checking that the condition on the righthand side is verified.

\footnotetext{
${ }^{3}$ We thank an anonymous reviewer for this remark.
} 
2) Riesz Basis: The polyharmonic B-splines $\left\{\beta_{\gamma}(\mathbf{x}-\right.$ k) $\}_{\mathbf{k} \in \mathbb{Z}^{N}}$ generate a Riesz basis, i.e., there exist two constants $0<C_{0}, C_{1}<\infty$ such that

$$
C_{0}\|c\|_{\ell_{2}} \leq\left\|\sum_{\mathbf{n}} c_{\mathbf{n}} \beta_{\gamma}(\mathbf{x}-\mathbf{n})\right\|_{L_{2}} \leq C_{1}\|c\|_{\ell_{2}} .
$$

This condition is equivalent to

$$
C_{0} \leq A_{\gamma}\left(e^{j \omega}\right) \leq C_{1}
$$

where $A_{\gamma}\left(e^{j \omega}\right)$ is the Fourier transform of the autocorrelation sequence $\left\langle\beta_{\gamma}(\cdot), \beta_{\gamma}(\cdot-\mathbf{n})\right\rangle$, i.e.,

$$
\begin{aligned}
A_{\gamma}\left(e^{j \boldsymbol{\omega}}\right) & =\sum_{\mathbf{n}}\left\langle\beta_{\gamma}(\cdot), \beta_{\gamma}(\cdot-\mathbf{n})\right\rangle \exp (-j\langle\boldsymbol{\omega}, \mathbf{n}\rangle) \\
& =\sum_{\mathbf{k}}\left|\hat{\beta}_{\gamma}(\boldsymbol{\omega}+2 \pi \mathbf{k})\right|^{2} .
\end{aligned}
$$

Using the convolution property of the polyharmonic B-spline, we can rewrite the autocorrelation filter as

$$
A_{\gamma}\left(e^{j \omega}\right)=\sum_{\mathbf{k}} \hat{\beta}_{2 \gamma}(\boldsymbol{\omega}+2 \pi \mathbf{k}) .
$$

Given that $A_{\gamma}\left(e^{j \omega}\right) \geq\left|\hat{\beta}_{\gamma}(\omega)\right|^{2}$ and that the continuous function $\left|\hat{\beta}_{\gamma}(\omega)\right|^{2}$ does not vanish inside $[-\pi, \pi]^{N}$, the existence of a lowerbound is trivial. The existence of the upperbound is also garanteed by the uniform convergence of the sum (18) as shown in Appendix I. As a sidenote, we would like to point out that there is a surprising connection between this autocorrelation filter and the so-called Epstein Zeta function which finds applications in crystallography (see [37]), in number theory (see [38]), and in quantum field theory (see [39]).

3) Orthonormal and Dual Flavors: The polyharmonic $\mathrm{B}$-splines $\beta_{\gamma}$ can be orthonormalized such that

$$
\left\langle\beta_{\gamma}^{\perp}(\mathbf{x}), \beta_{\gamma}^{\perp}(\mathbf{x}-\mathbf{n})\right\rangle=\delta_{\mathbf{n}}, \quad \text { for } \mathbf{n} \in \mathbb{Z}^{N} .
$$

The Fourier expression of $\beta_{\gamma}^{\perp}$ is given by

$$
\hat{\beta}_{\gamma}^{\perp}(\boldsymbol{\omega})=\frac{\hat{\beta}_{\gamma}(\boldsymbol{\omega})}{\sqrt{A_{\gamma}\left(e^{j \boldsymbol{\omega}}\right)}} .
$$

Analogously, one can define the dual polyharmonic B-spline as the unique function $\beta_{\gamma}^{\circ} \in \mathcal{V}\left(\beta_{\gamma}\right)$ that is biorthonormal to $\beta_{\gamma}(\mathbf{x})$. In the Fourier domain, this yields

$$
\hat{\beta}_{\gamma}^{\circ}(\boldsymbol{\omega})=\frac{\hat{\beta}_{\gamma}(\boldsymbol{\omega})}{A_{\gamma}\left(e^{j \boldsymbol{\omega}}\right)} .
$$

The dual splines are important since they allow us to specify the orthogonal projection of an $L^{2}$-function $f(\mathbf{x})$ onto $\mathcal{V}\left(\beta_{\gamma}\right)$; that is, the function of $\mathcal{V}\left(\beta_{\gamma}\right)$ that approximates $f(\mathbf{x})$ "best." Specifically, the projection $\mathcal{P} f(\mathbf{x})$ can be written equivalently as

$$
\begin{aligned}
\mathcal{P} f(\mathbf{x}) & =\sum_{\mathbf{n}}\left\langle f(\cdot), \beta_{\gamma}^{\circ}(\cdot-\mathbf{n})\right\rangle \beta_{\gamma}(\mathbf{x}-\mathbf{n}) \\
& =\sum_{\mathbf{n}}\left\langle f(\cdot), \beta_{\gamma}^{\perp}(\cdot-\mathbf{n})\right\rangle \beta_{\gamma}^{\perp}(\mathbf{x}-\mathbf{n}) \\
& =\sum_{\mathbf{n}}\left\langle f(\cdot), \beta_{\gamma}(\cdot-\mathbf{n})\right\rangle \beta_{\gamma}^{\circ}(\mathbf{x}-\mathbf{n}) .
\end{aligned}
$$

4) Spatial Decay: Unlike traditional B-splines (1-D or the tensor product extension) of integer order, the polyharmonic B-splines $(N \geq 2)$ are not compactly supported. Rabut has shown that the elementary polyharmonic B-splines decay like $O\left(1 /\|\mathbf{x}\|^{N+2}\right)$ as $\|\mathbf{x}\| \rightarrow \infty$. The proof in [35, Th. 2] is quite technical but can be extended. First, when $\gamma$ is not an even integer, the spatial decay becomes $O\left(1 /\|\mathrm{x}\|^{N+\min (2, \gamma)}\right)$. Second, for the isotropic polyharmonic B-splines, the new discretization of the Laplacian operator improves the smoothness of $\hat{\beta}_{\gamma}(\boldsymbol{\omega})$ around $\omega=0$ by 2 orders, which, in turn, increases the spatial decay of $\beta_{\gamma}(\mathbf{x})$ by two orders, at least for $\gamma$ sufficiently large. Therefore, we obtain a $O\left(1 /\|\mathbf{x}\|^{N+\min (4, \gamma)}\right)$ decay. This faster decay property is another indication that the isotropic basis functions are better localized.

5) Asymptotic Convergence: As a result of the central limit theorem and Proposition 2, we also know that the isotropic polyharmonic B-spline tend to the following isotropic Gaussian as $\gamma$ increases

$$
\beta_{\gamma}(\mathbf{x}) \approx\left(\frac{6}{\pi \gamma}\right)^{N / 2} \exp \left(-\frac{6\|\mathbf{x}\|^{2}}{\gamma}\right)
$$

whose standard deviation is $\sigma=\sqrt{\gamma / 12}$. The normalized squared difference between $\beta_{\gamma}$ and its Gaussian limiting function is below 5\% for $\gamma \geq 3$. For the case $\gamma=5$ of Fig. 2(b), the difference barely reaches $3 \%$.

Consequently, the order provides a tuning parameter for the size of the support, allowing us to search for an optimal tradeoff between spatial and spectral selectivity. Due to the convergence to a Gaussian, the isotropic polyharmonic B-splines tend to be asymptotically optimally localized in the sense of the Heisenberg uncertainty principle. More precisely, the product of their spatial and spectral bandwidth, defined as

$$
S_{\beta_{\gamma}} S_{\hat{\beta}_{\gamma}}, \quad \text { with } S_{\beta_{\gamma}}=\left(\frac{\int\|\tau\|^{2}\left|\beta_{\gamma}(\boldsymbol{\tau})\right|^{2} \mathrm{~d} \boldsymbol{\tau}}{\int\left|\beta_{\gamma}(\boldsymbol{\tau})\right|^{2} \mathrm{~d} \boldsymbol{\tau}}\right)^{1 / 2} .
$$

reaches the minimum $1 / 2$ as $\gamma$ increases.

6) Order of Approximation: Similar to classical B-splines, polyharmonic B-splines can approximate a given (well-behaved) function $f(\mathbf{x})$ to any required accuracy by projecting it onto a rescaled spline space with step size $h$. This property is related to the rate of decrease when the sampling grid gets finer of the approximation error between $f(\mathbf{x})$, where $f(\mathbf{x})$ and its $\gamma$ th derivative are in $L_{2}$, and the best polyharmonic B-spline representation

$$
\inf _{c(\mathbf{n})}\left\|f(\mathbf{x})-\sum_{\mathbf{n}} c(\mathbf{n}) \beta_{\gamma}(\mathbf{x} / h-\mathbf{n})\right\|_{L_{2}} \leq \text { Const } \times h^{\gamma}
$$

where the constant depends on $f$ and $\gamma$, but not on $h$. Given their Fourier definition, it is easy to show that

$$
\forall \mathbf{k} \in \mathbb{Z}^{N} \backslash\{0\}, \quad \hat{\beta}_{\gamma}(2 \pi \mathbf{k}+\boldsymbol{\omega})=O\left(\|\boldsymbol{\omega}\|^{\gamma}\right), \quad \text { for } \boldsymbol{\omega} \rightarrow \mathbf{0}
$$

which implies that the order of approximation for polyharmonic B-splines corresponds to $\gamma$ [40], [41], thus justifying our terminology and notation for the $\gamma$ th-order polyharmonic B-spline $\beta_{\gamma}$. 

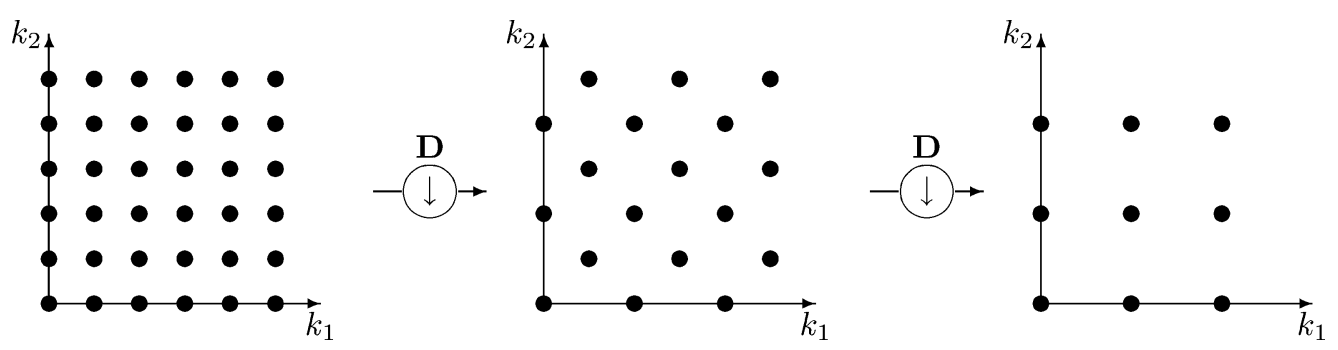

Fig. 3. Quincunx subsampling scheme for two iterations.

\section{MultiResolution AnAlysis}

In this section, we investigate the multiresolution properties of the polyharmonic B-splines. Madych [42], [43] showed already that certain polyharmonic splines are perfectly valid scaling functions. Micchelli et al. [44] constructed pre-wavelets from (elliptic) polyharmonic B-splines for dyadic subsampling schemes. Here we will start from the isotropic polyharmonic B-splines to build semi-orthogonal wavelet bases. First, we briefly show that isotropic polyharmonic B-splines are admissible scaling functions that satisfy a whole variety of scaling relations. We then concentrate on the 2-D quincunx subsampling scheme. When necessary, we will emphasize instances where we encounter an important difference between the elementary polyharmonic B-splines and the isotropic ones.

\section{A. Scaling Function and Scaling Relations}

We want to define a dilation matrix $\mathbf{M}$ that maps every point $\mathbf{k} \in \mathbb{Z}^{N}$ to a subset of $\mathbb{Z}^{N}$. Therefore, we introduce $\mathbf{M}$ as a matrix of size $N \times N$ that contains only integer elements and with $|\operatorname{det} \mathbf{M}| \geq 2$. Another, more technical, requirement is that all eigenvalues of $\mathbf{M}$ should be strictly greater than 1, i.e., $\mathbf{M}$ should be a dilation in all directions [45].

We now recall the approximation space spanned by the polyharmonic B-splines $\mathcal{V}\left(\beta_{\gamma}\right)$, as defined in (8). Similarly, we consider the approximation space $\mathcal{V}_{\mathbf{M}}$ at a finer resolution $\mathbf{M}$ as

$$
\mathcal{V}_{\mathbf{M}}=\operatorname{span}_{\mathbf{n} \in \mathbb{Z}^{N}}\left\{\beta_{\gamma}(\mathbf{M x}-\mathbf{n})\right\}
$$

where we will further elaborate on the admissible choices of $\mathbf{M}$ later on. The idea is to generate a sequence of embedded subspaces of $L_{2}\left(\mathbb{R}^{2}\right)$

$$
\cdots \mathcal{V}_{\mathbf{M}^{-1}} \subset \mathcal{V}_{\mathbf{M}^{0}} \subset \mathcal{V}_{\mathbf{M}} \subset \mathcal{V}_{\mathbf{M}^{2}} \subset \cdots L_{2} .
$$

Mallat [1] defined the minimal requirements that a scaling function needs to satisfy to generate an MRA. These requirements are functionally equivalent to: 1 ) Riesz conditions to ensure that we have shift-invariant subspaces, 2) Partition of unity to guarantee the convergence $\lim _{i \rightarrow \infty} \mathcal{V}_{\mathbf{M}^{i}}=L_{2}$, and 3) Scaling relation for $\mathbf{M}$. The first two properties have already been proven in the previous section. The scaling relation brings along the space inclusion property, i.e., it expresses $\beta_{\gamma}$ in the finer resolution space

$$
\beta_{\gamma}(\mathbf{x})=\sum_{\mathbf{n}} b_{\gamma}(\mathbf{n}) \beta_{\gamma}(\mathbf{M x}-\mathbf{n})
$$

or, equivalently, in the Fourier domain

$$
B_{\gamma}\left(e^{j \boldsymbol{\omega}}\right)=|\operatorname{det} \mathbf{M}| \frac{\hat{\beta}_{\gamma}\left(\mathbf{M}^{\mathrm{T}} \boldsymbol{\omega}\right)}{\hat{\beta}_{\gamma}(\boldsymbol{\omega})} .
$$

In order to obtain a valid scaling filter $b(\mathbf{n})$, the respective denominators of $\hat{\beta}_{\gamma}\left(\mathbf{M}^{\mathrm{T}} \boldsymbol{\omega}\right)$ and $\hat{\beta}_{\gamma}(\boldsymbol{\omega})$ in (29) need to cancel each other up to a scalar factor. Due to the isotropic denominator $\|\boldsymbol{\omega}\|^{\gamma}$ of the polyharmonic B-splines, $\mathbf{M}$ may correspond to any similarity transform, i.e., any rotation or symmetry combined with a dilation $|\operatorname{det} \mathbf{M}| \geq 2$. In particular, in two dimensions, the dilation matrix can be chosen

$$
\mathbf{M}=\left[\begin{array}{cc}
a & b \\
-b & a
\end{array}\right], \quad \text { or } \quad \mathbf{M}=\left[\begin{array}{cc}
a & b \\
b & -a
\end{array}\right]
$$

for $a$ and $b$ being integer and $a^{2}+b^{2} \geq 2$. It is interesting to mention that an MRA using the traditional tensor-product B-splines is much more restrictive, i.e., it requires the dilation matrix to be separable (corresponding to an integer scaling along each dimension).

Now, we can define the wavelet space $\mathcal{W}_{\mathrm{M}^{i}}$ uniquely as the orthogonal complement of $\mathcal{V}_{\mathbf{M}^{i}}$ in $\mathcal{V}_{\mathbf{M}^{i+1}}: \mathcal{V}_{\mathbf{M}^{i}} \oplus \mathcal{W}_{\mathbf{M}^{i}}=$ $\mathcal{V}_{\mathbf{M}^{i+1}}$. Finally, it is well-known that there exist $m=|\operatorname{det} \mathbf{M}|-$ 1 wavelets $\psi_{(1)}, \ldots, \psi_{(m)}$, that span the residual spaces $\mathcal{W}_{\mathbf{M}^{i}}$

$$
\mathcal{W}_{\mathbf{M}^{i}}=\operatorname{span}_{\mathbf{n} \in \mathbb{Z}^{N}}\left\{\psi_{(1)}\left(\mathbf{M}^{i} \mathbf{x}-\mathbf{n}\right), \ldots, \psi_{(m)}\left(\mathbf{M}^{i} \mathbf{x}-\mathbf{n}\right)\right\} .
$$

Many desirable mathematical properties of the wavelets, for instance, the number of vanishing moments, are directly related to the order $\gamma$ of the polyharmonic scaling function [12], [13].

\section{B. Quincunx Multiresolution Analysis}

For the remaining part of this paper, we focus on the 2-D quincunx dilation matrix, which is an interesting configuration for image processing (for which the traditional tensor product B-splines cannot be applied). As already mentioned in the introduction, the quincunx scheme provides a slower progression through scale than the traditional dyadic subsampling scheme. In addition, the wavelet space is spanned by only one wavelet, which simplifies its design and application.

The quincunx subsampling scheme, depicted in Fig. 3, can be represented by several possible dilation matrices [45]-[47]. For image processing, the most interesting one is based on a symmetry [17], [18], [48], [49] and is given by

$$
\mathbf{D}=\left[\begin{array}{cc}
1 & 1 \\
1 & -1
\end{array}\right]
$$

For an even number of iterations, the subsampled grid exactly coincides with the original cartesian grid at a twice coarser resolution, i.e., two subsequent scale reductions correspond to $\mathbf{D}^{2}=$ $2 \mathbf{I}$. As expected, the wavelet space is spanned by $|\operatorname{det} \mathbf{D}|-1=1$ wavelet. 


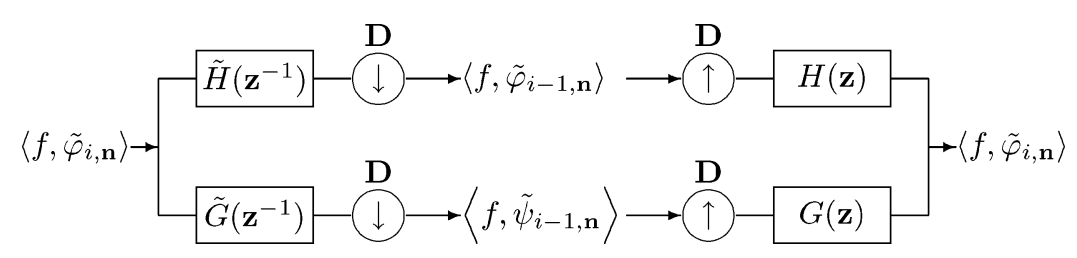

Fig. 4. Analysis-synthesis filterbank for the 2-D quincunx wavelet transform.

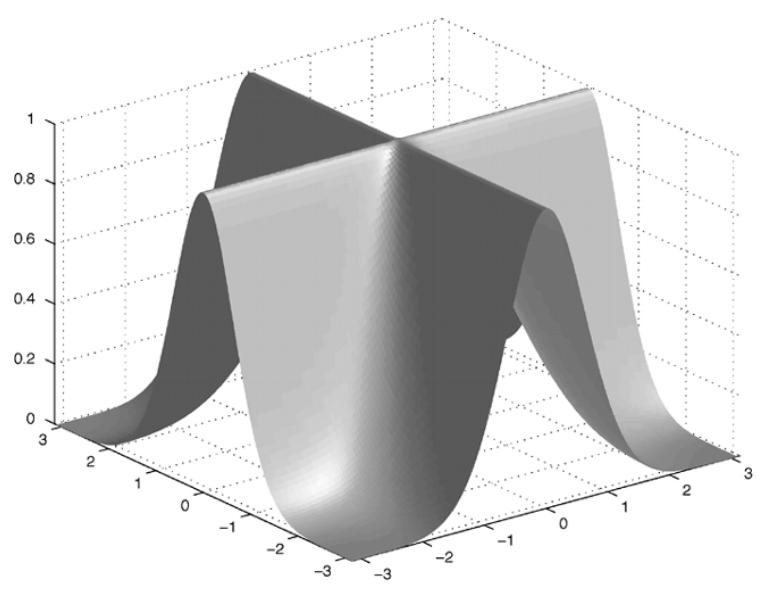

(a)

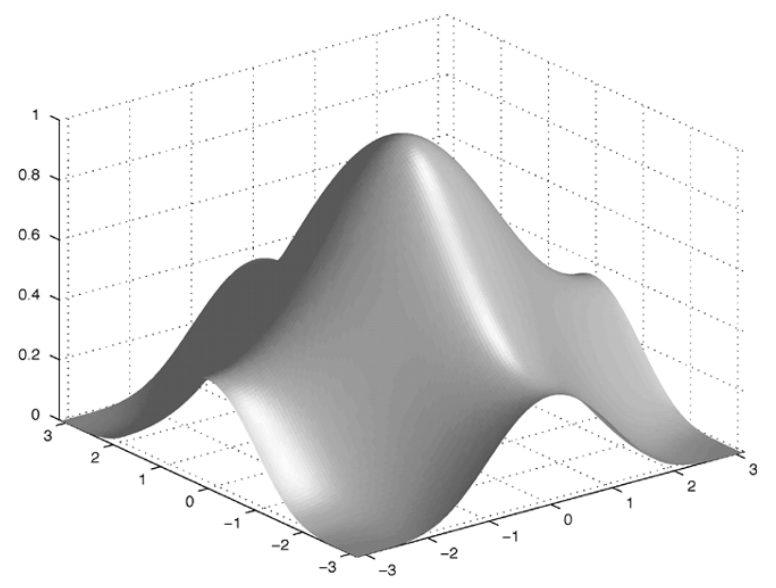

(b)

Fig. 5. Scaling filter $B_{\gamma}\left(e^{j \omega}\right) / 2$ associated with the quincunx dilation matrix for $\gamma=5$. (a) Elementary polyharmonic B-splines. (b) Isotropic polyharmonic B-splines.

In Fig. 4, we show the wavelet transform algorithm for one iteration. We introduce the following notation: $\varphi_{i, \mathbf{n}}(\mathbf{x})=$ $\sqrt{2^{i}} \varphi\left(\mathbf{D}^{i} \mathbf{X}-\mathbf{n}\right)$ and $\tilde{\varphi}_{i, \mathbf{n}}$ for the synthesis and analysis basis functions, respectively; $\psi_{i, \mathbf{n}}(\mathbf{x})=\sqrt{2^{i}} \psi\left(\mathbf{D}^{i} \mathbf{x}-\mathbf{n}\right)$ and $\tilde{\psi}_{i, \mathbf{n}}$ for the wavelets. The approximation of a function $f(\mathbf{x})$ at scale $i$ can be written as

$$
\begin{aligned}
\sum_{\mathbf{n}}\left\langle f, \tilde{\varphi}_{i, \mathbf{n}}\right\rangle \varphi_{i, \mathbf{n}}(\mathbf{x})=\sum_{\mathbf{n}}\langle & \left.f, \tilde{\varphi}_{i-1, \mathbf{n}}\right\rangle \varphi_{i-1, \mathbf{n}}(\mathbf{x}) \\
& +\sum_{\mathbf{n}}\left\langle f, \tilde{\psi}_{i-1, \mathbf{n}}\right\rangle \psi_{i-1, \mathbf{n}}(\mathbf{x}) .
\end{aligned}
$$

For an efficient filterbank implementation, one directly works with the coefficients $c_{(i)}(\mathbf{n})=\left\langle f, \tilde{\varphi}_{i, \mathbf{n}}\right\rangle$ and $d_{(i)}(\mathbf{n})=$ $\left\langle f, \tilde{\psi}_{i, \mathbf{n}}\right\rangle$ and computes the coefficients at the next coarser scale by filtering and downsampling. The scaling and wavelet filters are $\tilde{H}$ and $\tilde{G}$ on the analysis side, and $H$ and $G$ on the synthesis side, respectively. The conditions for perfect reconstruction that need to be satisfied by these filters are

$$
\begin{aligned}
\tilde{H}\left(\mathbf{z}^{-1}\right) H(\mathbf{z})+\tilde{G}\left(\mathbf{z}^{-1}\right) G(\mathbf{z}) & =2 \\
\tilde{H}\left(\mathbf{z}^{-1}\right) H(-\mathbf{z})+\tilde{G}\left(\mathbf{z}^{-1}\right) G(-\mathbf{z}) & =0 .
\end{aligned}
$$

\section{Polynomial Polyharmonic B-Spline Wavelets}

In this section, we will follow the design procedure of [50], where the wavelet is selected orthogonal to all the in- teger-shifted versions of the scaling function. This leads to a wavelet transform that is usually refered to as "semi-orthogonal." A direct consequence of this strategy is that the wavelet spaces are orthogonal to each other: $\mathcal{W}_{\mathrm{M}^{i}} \perp \mathcal{W}_{\mathrm{M}^{j}}$, for $i \neq j$.

Since we will derive all our scaling functions and wavelets from the isotropic polyharmonic B-splines, we first show the scaling relation satisfied by these splines for the quincunx dilation matrix. By using (29) with the quincunx dilation matrix (32), we obtain (36), shown at the bottom of the page. Fig. 5(b) shows the frequency response of this filter for order $\gamma=5$. Also shown is the scaling filter for the elementary polyharmonic B-splines, which exhibits a much less favorable "cross-like" structure and this for any order $\gamma$. This is in contrast with the isotropic filter which becomes more and more Gaussian-like as $\gamma$ increases.

1) Polyharmonic B-Spline Wavelet Transform: As the first example, we select the isotropic polyharmonic B-spline $\beta_{\gamma}$ as scaling function $\varphi$. We look for the associated wavelet $\psi$, included in the function space $\mathcal{V}_{\mathrm{D}^{0}}$ at a finer scale

$$
\psi\left(\mathbf{D}^{-1} \mathbf{x}\right)=\sum_{\mathbf{n}} w(\mathbf{n}) \beta_{\gamma}(\mathbf{x}-\mathbf{n})
$$

that also satisfies the orthogonality condition. Indeed, a necesary condition for $\mathcal{W}_{\mathbf{D}^{-1}} \perp \mathcal{V}_{\mathbf{D}^{-1}}$ is

$$
\left\langle\psi\left(\mathbf{D}^{-1} \mathbf{x}\right), \beta_{\gamma}\left(\mathbf{D}^{-1} \mathbf{x}-\mathbf{n}\right)\right\rangle=0 .
$$

$$
B_{\gamma}\left(e^{j \omega}\right)=2^{1-\gamma / 2}\left(\frac{\sin ^{2}\left(\frac{\omega_{1}+\omega_{2}}{2}\right)+\sin ^{2}\left(\frac{\omega_{1}-\omega_{2}}{2}\right)-\frac{2}{3} \sin ^{2}\left(\frac{\omega_{1}+\omega_{2}}{2}\right) \sin ^{2}\left(\frac{\omega_{1}-\omega_{2}}{2}\right)}{\sin ^{2}\left(\frac{\omega_{1}}{2}\right)+\sin ^{2}\left(\frac{\omega_{2}}{2}\right)-\frac{2}{3} \sin ^{2}\left(\frac{\omega_{1}}{2}\right) \sin ^{2}\left(\frac{\omega_{2}}{2}\right)}\right)^{\gamma / 2}
$$


This condition can be expressed equivalently in the $z$-domain as

$$
W(\mathbf{z}) B_{\gamma}\left(\mathbf{z}^{-} \mathbf{1}\right) A_{\gamma}(\mathbf{z})+W(-\mathbf{z}) B_{\gamma}(-\mathbf{z}) A_{\gamma}\left(-\mathbf{z}^{-} \mathbf{1}\right)=0 .
$$

It can be shown that the general solution of this equation is

$$
W(\mathbf{z})=-z_{1}^{-1} Q\left(\mathbf{z}^{\mathbf{D}}\right) B_{\gamma}\left(-\mathbf{z}^{-1}\right) A_{\gamma}(-\mathbf{z})
$$

where $Q(\mathbf{z})$ is an arbitrary polynomial in $\mathbf{z}$. Here, $\mathbf{z}^{\mathbf{D}}$ is a shortcut notation for $\left(z_{1} z_{2}, z_{1} z_{2}^{-1}\right)$; the Fourier transform of $Q\left(\mathbf{z}^{\mathbf{D}}\right)$ corresponds to $Q\left(e^{j \mathbf{D}^{\mathrm{T}} \boldsymbol{\omega}}\right)$. The most obvious choice is $Q(\mathbf{z})=1$, which gives us

$$
\hat{\psi}\left(\mathbf{D}^{\mathrm{T}} \boldsymbol{\omega}\right)=\frac{W\left(e^{j \omega}\right)}{2} \hat{\beta}_{\gamma}(\boldsymbol{\omega})
$$

with $W\left(e^{j \boldsymbol{\omega}}\right)=-e^{-j \omega_{1}} B_{\gamma}\left(e^{-j(\boldsymbol{\omega}+\boldsymbol{\pi})}\right) A_{\gamma}\left(e^{j(\boldsymbol{\omega}+\boldsymbol{\pi})}\right)$, where we used the property $B_{\gamma}\left(\mathbf{z}^{-\mathbf{1}}\right)=B_{\gamma}(\mathbf{z})$. This wavelet is referred to as the "isotropic polyharmonic B-spline wavelet."

Of course, the polynomial $Q(\mathbf{z})$ needs to satisfy certain properties to obtain suitable wavelets that form a Riesz basis. This is expressed by the following theorem, which is valid for any scaling function and the quincunx subsampling scheme.

Theorem 1: Let $\varphi(\mathbf{x})$ be a valid scaling function for the quincunx subsampling scheme. Specifically, it has a scaling filter $B_{\gamma}\left(e^{j \omega}\right)$ and it forms a Riesz basis, i.e., the autocorrelation filter $A_{\gamma}\left(e^{j \omega}\right)$ is bounded by two constants $0<C_{0} \leq A_{\gamma}\left(e^{j \omega}\right) \leq$ $C_{1}<\infty$. Then, the wavelet given by

$$
\hat{\psi}\left(\mathbf{D}^{\mathrm{T}} \boldsymbol{\omega}\right)=\frac{W\left(e^{j \boldsymbol{\omega}}\right)}{2} \hat{\varphi}(\boldsymbol{\omega})
$$

with $W\left(e^{j \boldsymbol{\omega}}\right)=-e^{-j \omega_{1}} Q\left(e^{j \mathbf{D}^{\mathrm{T}} \boldsymbol{\omega}}\right) B_{\gamma}\left(e^{j(\boldsymbol{\omega}+\boldsymbol{\pi})}\right) A_{\gamma}\left(e^{j(\boldsymbol{\omega}+\boldsymbol{\pi})}\right)$ forms a Riesz basis as well, as long as $\left|Q\left(e^{j \omega}\right)\right|^{2}$ is also bounded by two constants $0<C_{0}^{\prime} \leq\left|Q\left(e^{j \omega}\right)\right|^{2} \leq C_{1}^{\prime}<\infty$.

Proof: We compute the autocorrelation filter of the wavelet $\hat{\psi}(\boldsymbol{\omega})$

$$
\begin{aligned}
R\left(e^{j \boldsymbol{\omega}}\right)= & \sum_{\mathbf{k}}|\hat{\psi}(\boldsymbol{\omega}+2 \pi \mathbf{k})|^{2} \\
= & \frac{\left|W\left(e^{j \mathbf{D}^{-\mathrm{T}} \boldsymbol{\omega}}\right)\right|^{2}}{4} A_{\gamma}\left(e^{j \mathbf{D}^{-\mathrm{T}} \boldsymbol{\omega}}\right) \\
& +\frac{\left|W\left(e^{j\left(\mathbf{D}^{-\mathrm{T}} \boldsymbol{\omega}+\boldsymbol{\pi}\right)}\right)\right|^{2}}{4} A_{\gamma}\left(e^{j\left(\mathbf{D}^{-\mathrm{T}} \boldsymbol{\omega}+\boldsymbol{\pi}\right)}\right) \\
= & A_{\gamma}\left(e^{j \mathbf{D}^{-\mathrm{T}} \boldsymbol{\omega}}\right) A_{\gamma}\left(e^{j\left(\mathbf{D}^{-\mathrm{T}} \boldsymbol{\omega}+\boldsymbol{\pi}\right)}\right) \\
& \times\left|Q\left(e^{j \boldsymbol{\omega}}\right)\right|^{2}\left(\frac{\left|B_{\gamma}\left(e^{j \mathbf{D}^{-\mathrm{T}} \boldsymbol{\omega}}\right)\right|^{2}}{4} A_{\gamma}\left(e^{j \mathbf{D}^{-\mathrm{T}} \boldsymbol{\omega}}\right)\right. \\
& \left.+\frac{\left|B_{\gamma}\left(e^{j\left(\mathbf{D}^{-\mathrm{T}} \boldsymbol{\omega}+\boldsymbol{\pi}\right)}\right)\right|^{2}}{4} A_{\gamma}\left(e^{j\left(\mathbf{D}^{-\mathrm{T}} \boldsymbol{\omega}+\boldsymbol{\pi}\right)}\right)\right) \\
= & A_{\gamma}\left(e^{j \mathbf{D}^{-\mathrm{T}} \boldsymbol{\omega}}\right) A_{\gamma}\left(e^{j\left(\mathbf{D}^{-\mathrm{T}} \boldsymbol{\omega}+\boldsymbol{\pi}\right)}\right) A_{\gamma}\left(e^{j \boldsymbol{\omega}}\right)\left|Q\left(e^{j \boldsymbol{\omega}}\right)\right|^{2} .
\end{aligned}
$$

Consequently, the wavelet forms a Riesz basis if we have $0<$ $C_{0}^{\prime} \leq\left|Q\left(e^{j \omega}\right)\right|^{2} \leq C_{1}^{\prime}<\infty$.

The construction of the wavelet that leads to (40) describes the synthesis side of the wavelet transform. The complete transform, as indicated by (33), also requires the analysis scaling function and wavelet. To obtain the corresponding analysis funtions, we compute the dual scaling function that is given by

$$
\hat{\tilde{\varphi}}(\boldsymbol{\omega})=\hat{\beta}_{\gamma}^{\circ}(\boldsymbol{\omega})=\frac{\hat{\beta}_{\gamma}(\boldsymbol{\omega})}{A_{\gamma}\left(e^{j \boldsymbol{\omega}}\right)}
$$

and we automatically obtain the associated dual scaling filter

$$
B_{\gamma}^{\circ}\left(e^{j \omega}\right)=\frac{A_{\gamma}\left(e^{j \omega}\right)}{A_{\gamma}\left(e^{j \mathbf{D}^{\mathrm{T}} \boldsymbol{\omega}}\right)} B_{\gamma}\left(e^{j \omega}\right) .
$$

In a way similar to (42), we find the autocorrelation of the polyharmonic B-spline wavelet $R_{\gamma}\left(e^{j \boldsymbol{\omega}}\right)$. Then, the dual wavelet can be concisely defined as

$$
\hat{\tilde{\psi}}(\boldsymbol{\omega})=\hat{\psi}^{\circ}(\boldsymbol{\omega})=\frac{\hat{\psi}(\boldsymbol{\omega})}{R_{\gamma}\left(e^{j \omega}\right)} .
$$

The corresponding dual wavelet relation

$$
\hat{\psi}^{\circ}\left(\mathbf{D}^{\mathrm{T}} \boldsymbol{\omega}\right)=\frac{W^{\circ}\left(e^{j \boldsymbol{\omega}}\right)}{2} \hat{\beta}_{\gamma}^{\circ}(\boldsymbol{\omega})
$$

is obtained from (40) with

$$
W^{\circ}\left(e^{j \boldsymbol{\omega}}\right)=-e^{-j \omega_{1}} \frac{B_{\gamma}^{\circ}\left(e^{-j(\boldsymbol{\omega}+\boldsymbol{\pi})}\right)}{A_{\gamma}\left(e^{j \mathrm{D}^{T} \boldsymbol{\omega}}\right) .}
$$

In practice, an efficient filterbank implementation will directly rely on the scaling and wavelet filters to process the coefficients. Table I lists all the filters and functions involved for the isotropic polyharmonic B-spline wavelet transform. Fig. 7 depicts the respective scaling functions and wavelets in the spatial domain.

The dual isotropic polyharmonic B-spline wavelet transform can be found by interchanging the functions and filters between the analysis and synthesis part. Depending on the application, it might be desirable to put the B-spline either on the analysis or the synthesis side. The main feature of this wavelet decomposition is the excellent space-frequency localization of the B-spline scaling function and its wavelet. We already indicated that the isotropic polyharmonic B-splines tend to a Gaussian function as the order $\gamma$ increases. Similarly, the associated wavelets tend to modulated Gaussians, also known as Gabor functions. In Appendix II, we give a proof of this convergence. In Fig. 6, we show the tiling of these wavelets in the frequency domain for eight consecutive iterations.

Indeed, Gabor wavelet-like decompositions have been found to be useful in many applications, as shown by the vast literature dealing with these functions [51]-[57]. Some examples include edge detection [58], [59], segmentation, texture analysis [60], modeling of primate's visual systems [61]-[63], statistical analysis of time-series (e.g., as in fMRI [64]), image analysis [65]-[67], hierarchical reconstruction [68], and so on. So, the refinement filters of the isotropic polyharmonic B-spline wavelet transform might be good candidates to build a proper 
TABLE I

OVERVIEW OF ISOTROPIC POLYHARMONIC B-SPLINE WAVELET TRANSFORM

\begin{tabular}{l||c|c}
\hline \hline & analysis & synthesis \\
\hline \hline scaling function & $\beta_{\gamma}^{\circ}(\mathbf{x}) \longleftrightarrow \frac{\hat{\beta}_{\gamma}(\boldsymbol{\omega})}{A_{\gamma}\left(e^{j \omega}\right)}$ & $\beta_{\gamma}(\mathbf{x})$ \\
scaling filter & $\tilde{H}(\mathbf{z})=B_{\gamma}^{\circ}(\mathbf{z})=\frac{A_{\gamma}(\mathbf{z})}{A_{\gamma}\left(\mathbf{z}^{\mathbf{D}}\right)} B_{\gamma}(\mathbf{z})$ & $H(\mathbf{z})=B_{\gamma}(\mathbf{z})$ \\
wavelet function & $\psi^{\circ}(\mathbf{x}) \longleftrightarrow \hat{\psi}^{\circ}(\boldsymbol{\omega})=\frac{\hat{\psi}(\boldsymbol{\omega})}{R_{\gamma}\left(e^{j \omega}\right)}$ & $\psi(\mathbf{x}) \longleftrightarrow \hat{\psi}(\boldsymbol{\omega})=\frac{W\left(e^{\left.j \mathbf{D}^{-\mathrm{T}} \boldsymbol{\omega}\right)}\right.}{2} \hat{\beta}_{\gamma}(\boldsymbol{\omega})$ \\
wavelet filter & $\tilde{G}(\mathbf{z})=W^{\circ}(\mathbf{z})=-z_{1}^{-1} \frac{B_{\gamma}^{\circ}\left(-\mathbf{z}^{-1}\right)}{A_{\gamma}(-\mathbf{z})}$ & $G(\mathbf{z})=W(\mathbf{z})=-z_{1}^{-1} B_{\gamma}\left(-\mathbf{z}^{-1}\right) A_{\gamma}(-\mathbf{z})$ \\
\hline \hline
\end{tabular}

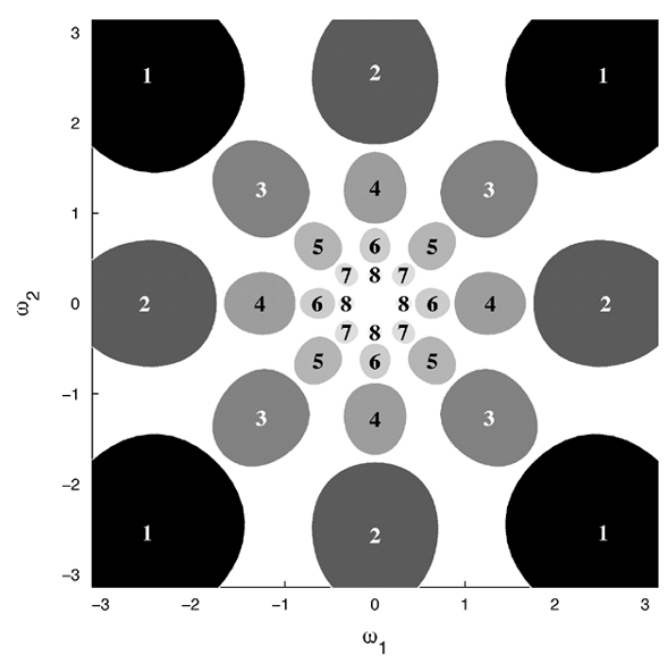

Fig. 6. Illustration of the tiling of the frequency domain for the polyharmonic B-spline wavelets of order $\gamma=5$ up to the eighth iteration. All contours shows their corresponding iteration number.

scale-space decomposition with a continuously tunable order parameter.

2) Orthonormal Polyharmonic B-Spline Wavelet Transform: Another important wavelet transform is the orthonormal one, in which the scaling function and wavelets are orthonormal with regard to their own shifts. Note that for this transform, there will be no difference between the isotropic polyharmonic B-splines and the elementary ones: Since both span the same space, their orthonormalized forms are equivalent. We note, however, that we have not yet seen these functions applied in the quincunx case, not to mention their fractional extensions.

The orthonormal polyharmonic B-spline is given by (20) and its corresponding scaling filter is

$$
B_{\gamma}^{\perp}\left(e^{j \omega}\right)=\sqrt{\frac{A_{\gamma}\left(e^{j \boldsymbol{\omega}}\right)}{A_{\gamma}\left(e^{j \mathbf{D}^{\mathrm{T}} \boldsymbol{\omega}}\right)}} B_{\gamma}\left(e^{j \boldsymbol{\omega}}\right) .
$$

Similarly, the orthonormal polyharmonic B-spline wavelet reads

$$
\hat{\psi}^{\perp}(\boldsymbol{\omega})=\frac{\hat{\psi}(\boldsymbol{\omega})}{\sqrt{R_{\gamma}\left(e^{j \omega}\right)}}
$$

with corresponding wavelet filter

$$
W^{\perp}\left(e^{j \boldsymbol{\omega}}\right)=-e^{-j \omega_{1}} B_{\gamma}^{\perp}\left(e^{j(\boldsymbol{\omega}+\boldsymbol{\pi})}\right) .
$$

Some examples of such functions are shown in Fig. 7(c)-(d).

In Appendix III, we prove the convergence of the orthonormal polyharmonic B-spline to the sinc function.
As for every linear orthonormal transform, the $L_{2}$-norm is conserved and white noise will remain white after transformation-a useful property for image denoising.

3) Generalized Polyharmonic B-Spline Wavelet Transforms: The B-spline, dual, and orthonormal flavor of the polyharmonic wavelet transforms are probably the most interesting candidates for applications. The design procedure for semi-orthogonal wavelet can also lead to other wavelets. In particular, the choice of $Q\left(\mathbf{z}^{\mathbf{D}}\right)$ in (39) is a degree of freedom that can be further explored. For example, we could select the interpolating polyharmonic B-spline at the synthesis side. This would eliminate the need of the initialization procedure that is needed to compute the initial values of the coefficients.

An interesting property of every polyharmonic wavelet is the behavior for low frequencies.

Proposition 3: The polyharmonic wavelets, obtained for any admissible choice of $Q\left(\mathbf{z}^{\mathbf{D}}\right)$, behave as the $\gamma / 2$ th iterate of the Laplacian operator for low frequencies.

Proof: We consider (39) and (40) for $\boldsymbol{\omega} \rightarrow \mathbf{0}$. The only term that tends to zero, and as such dominates the behavior of $\hat{\psi}(\boldsymbol{\omega})$ for $\boldsymbol{\omega} \rightarrow \mathbf{0}$, is $B_{\gamma}\left(e^{j(\boldsymbol{\omega}+\boldsymbol{\pi})}\right)$. Using (36), we can conclude that $\hat{\psi}(\boldsymbol{\omega}) \propto\|\boldsymbol{\omega}\|^{\gamma}$ for low frequencies.

This proposition shows how the operator that is related to the fundamental property of the polyharmonic B-splines gets transplanted to the wavelet functions.

In Fig. 8(b), we show an example wavelet decomposition of the "zoneplate" imageof (a). The subbands are organized in a way that is standard for the quincunx subsampling scheme, i.e., for odd iterations the odd lines are shifted by one pixel and then odd columns are subsampled (see, also, Section IV). The intensity values within each subband of Fig. 8(b) have been rescaled to improve visualization. In the first subband, we only capture high frequency components at the corners of the frequency sweep. In fact, the local spatial frequency at these corners is close to the center frequency of the corresponding Gabor atoms depicted in Fig. 6. As we progress through scale, the "sensitive" regions detected by the wavelet have lower spatial frequencies. Interestingly, at some juncture, we switch from "Gabor regime" to "Laplacian regime."

\section{IMPLEMENTATION OF THE POLYHARMONIC B-SPLINE WAVELET TRANSFORM}

All filters encountered in our construction are nonseparable and infinitely supported. Therefore, a spatial implementation based on truncation turns out to be difficult and costly. However, the proposed polyharmonic wavelet transform can be advantageously implemented in the Fourier domain using FFTs, 


\section{B-spline flavor}

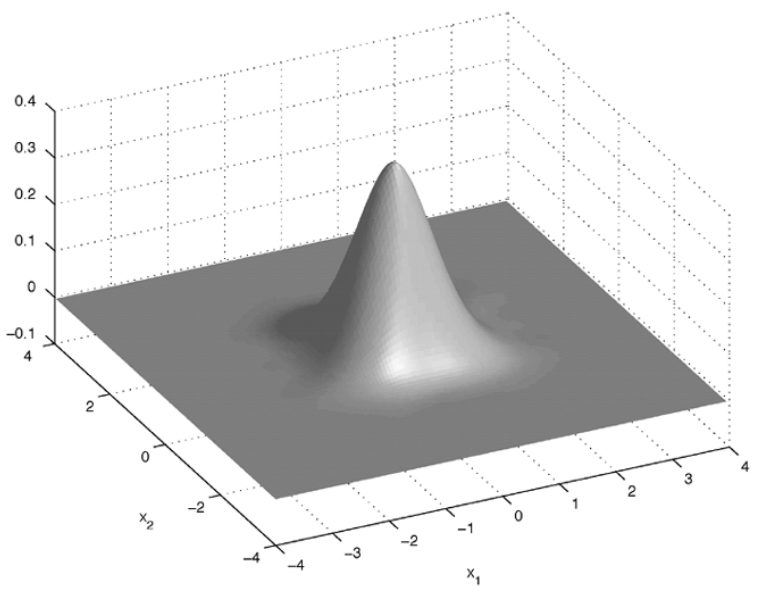

(a)

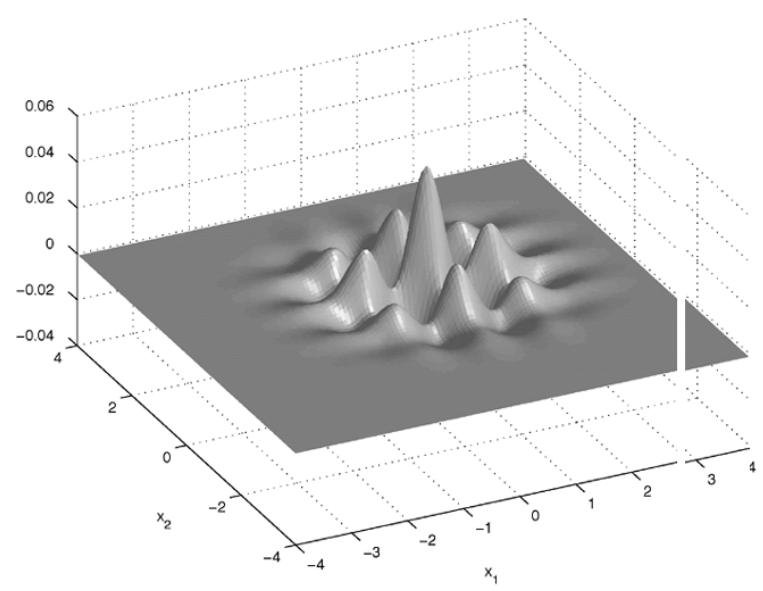

(b)

Orthonormal flavor

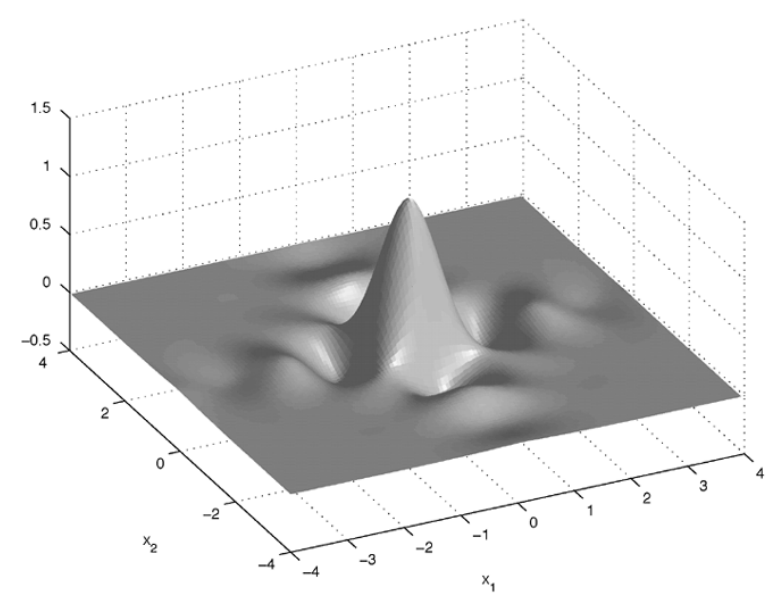

(c)

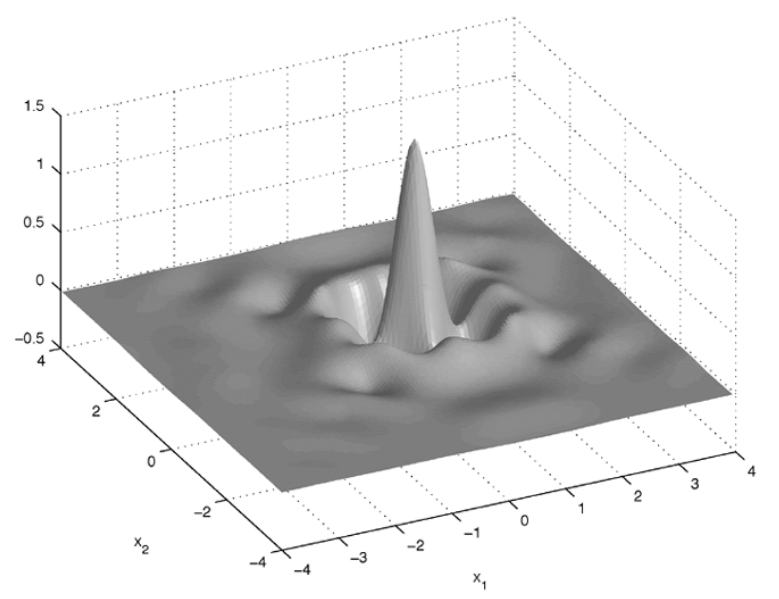

(d)

Dual flavor

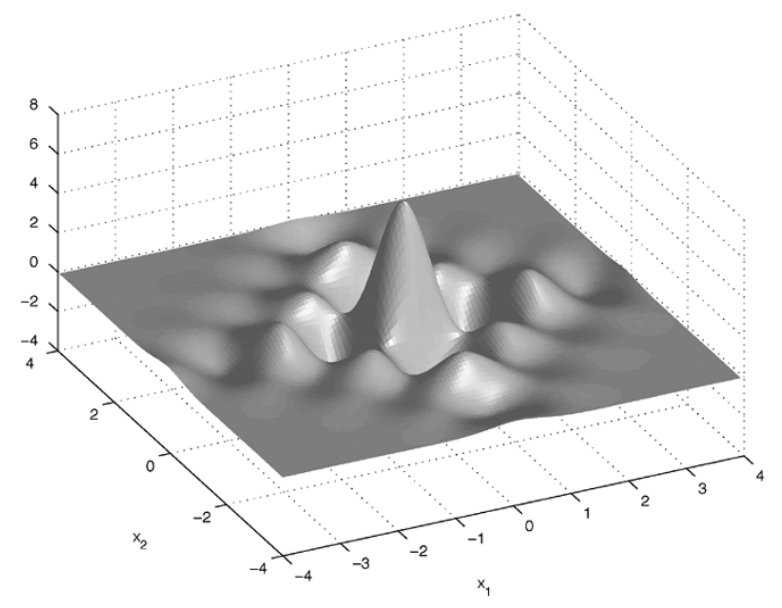

(e)

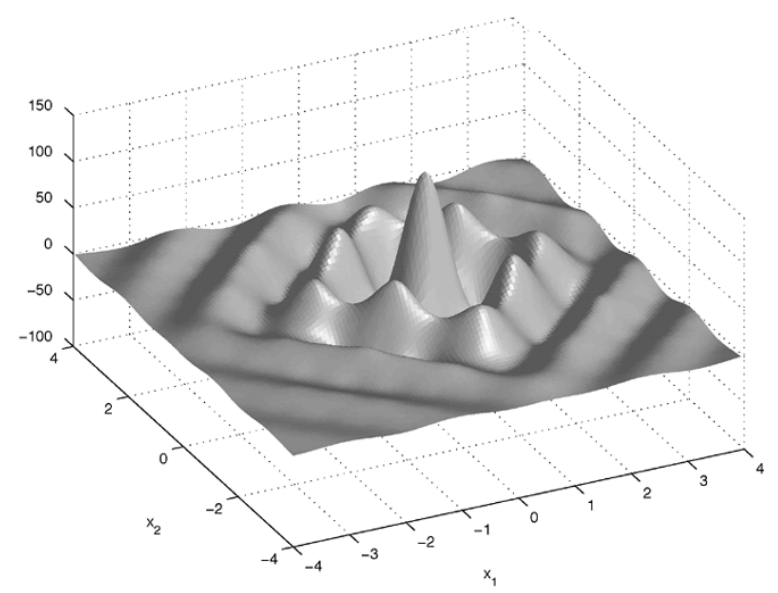

(f)

Fig. 7. Various brands of scaling functions and wavelets based on isotropic polyharmonic B-splines of order $\gamma=5.0$. (a) Isotropic polyharmonic B-spline. (b) Isotropic polyharmonic B-spline wavelet. (c) Orthonormalized polyharmonic B-spline. (d) Orthonormalized polyharmonic B-spline wavelet. (e) Dual isotropic polyharmonic B-spline. (f) Dual isotropic polyharmonic B-spline wavelet.

using the knowledge of the frequency response of the filters. Our Fourier-based implementation will also automatically take care of the boundaries by imposing periodic boundary conditions. Fourier-based implementations of the wavelet transform have been proposed before [22], [48], [49]. Using the same prin- ciple, we propose a slightly simplified version that is straightforward to implement and still performs very well.

To obtain the scaling and wavelet filters in the frequency domain, we need to compute the autocorrelation filter $A_{\gamma}\left(e^{j \omega}\right)$. This can be done in two dimensions by evaluating the sum of 
(a)

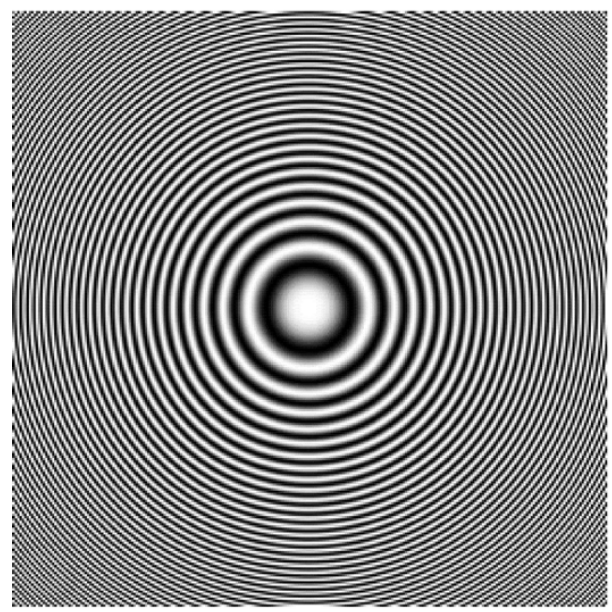

(c)

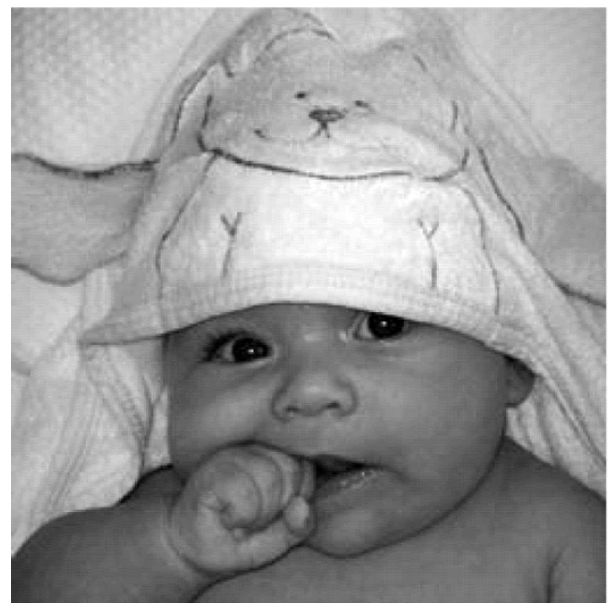

(b)

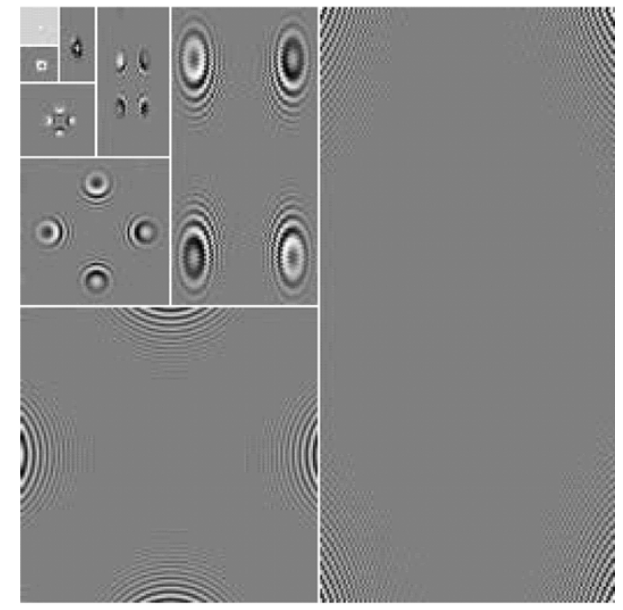

(d)

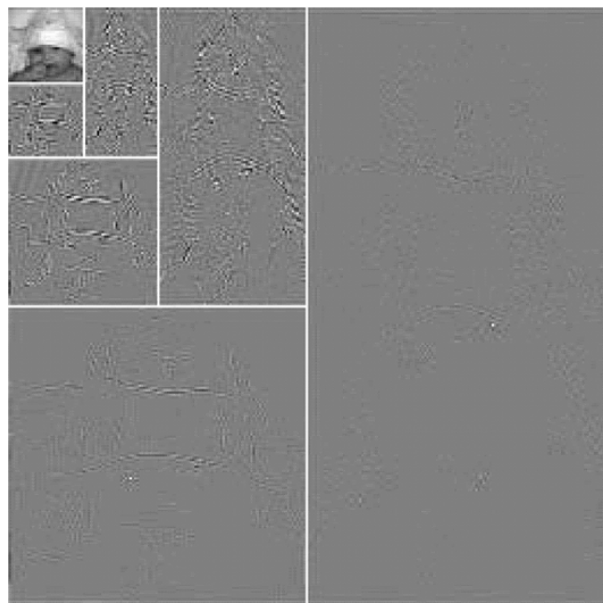

Fig. 8. (a) Test image "zoneplate." (b) Decomposition of "zoneplate" for eight iterations and order $\gamma=5$, B-splines at analysis. (c) Test image "Matteo." (d) Decomposition of "Matteo" after six iterations and order $\gamma=5$, orthonormal flavor.

(18) for a sufficient number of terms. Another approach is to use a numerical algorithm in the spatial domain (for example, see [69]). It is also possible to use a fastly converging method in the Fourier domain, as proposed in [70].

\section{A. Fast Fourier-Based Discrete Wavelet Transform}

The filterbank implementation of the wavelet transform directly deals with the coefficients $c_{(i)}(\mathbf{n})$ and $d_{(i)}(\mathbf{n})$, as defined before. However, all filters involved with the polyharmonic wavelet transform are infinitely supported. Because of this, and also because our filters are characterized in the frequency domain, we propose the use of a Fourier-based implementation, ensuring perfect reconstruction for any choice of the order $\gamma$.

1) Initialization: At initialization, the signal is characterized by the coefficients $c_{(0)}(\mathbf{n})=\left\langle f, \tilde{\varphi}_{0, \mathbf{n}}\right\rangle$, for a given support $S=\left\{\mathbf{n} \mid n_{1}, n_{2}=0, \ldots, M-1\right\}$. Under the assumption that we only have access to the sample values $f(\mathbf{n})$, the data needs to be prefiltered such that the interpolating condition is satisfied, i.e.,

$$
f(\mathbf{n})=\sum_{\mathbf{m}} c_{(0)}(\mathbf{m}) \beta_{\gamma}(\mathbf{n}-\mathbf{m}) .
$$

The proper interpolation prefilter is given by

$$
P\left(e^{j \boldsymbol{\omega}}\right)=\left(\sum_{\mathbf{k}} \hat{\beta}_{\gamma}(\boldsymbol{\omega}+2 \pi \mathbf{k})\right)^{-1}
$$

The same iterative numerical algorithm as used for the computation of the autocorrelation filter can be deployed to calculate this filter.

In practice, we first compute the Fourier coefficients of the data samples, for which we introduce the notation $F[\mathbf{k}]=F\left(e^{j 2 \pi \mathbf{k} / M}\right)=\sum_{\mathbf{n} \in S} f(\mathbf{n}) e^{-j 2 \pi\langle\mathbf{n}, \mathbf{k}\rangle / M}, \mathbf{k} \in S$. Note that the use of such a sampled Fourier representation corresponds to a periodic extension of the data in the spatial domain. The initial coefficients after prefiltering are

$$
C_{(0)}[\mathbf{k}]=F[\mathbf{k}] P[\mathbf{k}]
$$

2) Analysis: An efficient implementation of the wavelet transform in the Fourier domain for the quincunx subsampling scheme can be obtained by pooling together two levels of the decomposition tree. Fig. 9 shows a flowchart illustrating this approach. The indications $\mathcal{F}$ and $\mathcal{F}^{-1}$ mark where the data is, 


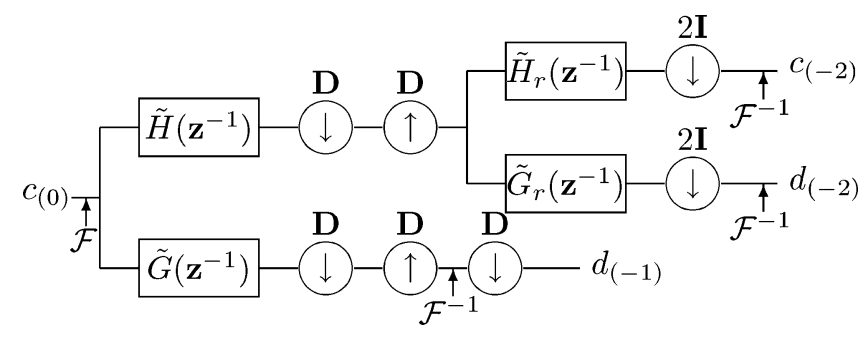

Fig. 9. Two levels of the quincunx wavelet decomposition stacked together. The symbols $\mathcal{F}$ and $\mathcal{F}^{-1}$ show where, respectively, the Fourier transform and the inverse Fourier transform is computed.

respectively, converted to and from the Fourier domain. The main steps of the algorithms are as follows.

1) The Fourier coefficients $C_{(0)}[\mathbf{k}]$ are filtered and then down- and upsampled, introducing redundancy in the Fourier domain. Taken together, such an operation results into

$$
\begin{aligned}
C_{(-1)}\left[\mathbf{k}^{\prime}\right]= & \frac{1}{2}\left(\tilde{H}\left[\mathbf{k}^{\prime}\right] C_{(0)}\left[\mathbf{k}^{\prime}\right]+\right. \\
& \left.\tilde{H}\left[\mathbf{k}^{\prime}+(M / 2, M / 2)\right] C_{(0)}\left[\mathbf{k}^{\prime}+(M / 2, M / 2)\right]\right) \\
D_{(-1)}[\mathbf{k}]= & \frac{1}{2}\left(\tilde{G}[\mathbf{k}] C_{(0)}[\mathbf{k}]+\right. \\
& \left.\tilde{G}[\mathbf{k}+(M / 2, M / 2)] C_{(0)}[\mathbf{k}+(M / 2, M / 2)]\right)
\end{aligned}
$$

where the index $\mathbf{k}^{\prime}$ for the lowpass filter can be limited to $S^{\prime}=\left\{\mathbf{n} \mid n_{1}=0, \ldots, M / 2-1 ; n_{2}=0, \ldots, M-1\right\}$. The easiest way to generate the highpass output $d_{(-1)}\left[\mathbf{k}^{\prime}\right]$ is by computing the inverse Fourier transform of $D_{(-1)}[\mathrm{k}]$ and performing the subsampling in the spatial domain. ${ }^{4}$

2) Next, the lowpass Fourier coefficients $C_{(-1)}\left[\mathbf{k}^{\prime}\right]$ are processed for the next iteration by the rotated filters, defined as $\tilde{H}_{r}[\mathbf{n}]=\tilde{H}[\mathbf{D n} \bmod (M, M)], \tilde{G}_{r}[\mathbf{n}]=$ $\tilde{G}[\mathbf{D n} \bmod (M, M)]$. So, we get

$$
\begin{aligned}
C_{(-2)}\left[\mathbf{k}^{\prime \prime}\right]= & \frac{1}{2}\left(\tilde{H}_{r}\left[\mathbf{k}^{\prime \prime}\right] C_{(-1)}\left[\mathbf{k}^{\prime \prime}\right]+\right. \\
& \left.\tilde{H}_{r}\left[\mathbf{k}^{\prime \prime}+(0, M / 2)\right] C_{(-1)}\left[\mathbf{k}^{\prime \prime}+(0, M / 2)\right]\right) \\
D_{(-2)}\left[\mathbf{k}^{\prime \prime}\right]= & \frac{1}{2}\left(\tilde{G}_{r}\left[\mathbf{k}^{\prime \prime}\right] C_{(-1)}\left[\mathbf{k}^{\prime \prime}\right]+\right. \\
& \left.\tilde{G}_{r}\left[\mathbf{k}^{\prime \prime}+(0, M / 2)\right] C_{(-1)}\left[\mathbf{k}^{\prime \prime}+(0, M / 2)\right]\right)
\end{aligned}
$$

where $\mathbf{k}^{\prime \prime} \in S^{\prime \prime}=\left\{\mathbf{n} \mid n_{1}, n_{2}=0, \ldots, M / 2-1\right\}$. The outputs $c_{(-2)}\left[\mathbf{k}^{\prime \prime}\right]$ and $d_{(-2)}\left[\mathbf{k}^{\prime \prime}\right]$ are now directly obtained by applying the inverse Fourier transform to $C_{(-2)}\left[\mathbf{k}^{\prime \prime}\right]$ and $D_{(-2)}\left[\mathbf{k}^{\prime \prime}\right]$. Depending on the number of iterations, the remaining lowpass signal can be kept in the Fourier domain and further decomposed. A convenient way to arrange the coefficients is shown in Fig. 8(b) and (d).

\footnotetext{
${ }^{4}$ It is also possible to exploit the redundancy of $D_{(-1)}[\mathbf{k}]$ in the Fourier do-
} main (see [22]).
The filters $\tilde{H}, \tilde{G}$, and their rotated versions $\tilde{H}_{r}, \tilde{G}_{r}$ are precomputed at the size of the original data and subsampled after each other iteration. We also silently assumed that the analysis filters are reversed, i.e., their discrete Fourier transform corresponds to $\tilde{H}[\mathbf{k}]=\tilde{H}\left(e^{-j 2 \pi \mathbf{k} / M}\right)$.

3) Synthesis: Using the same principles, one can obtain the synthesis algorithm as the flow graph transpose of the analysis algorithm. Again, all filters are precomputed.

\section{B. Benchmark}

The proposed algorithm can be translated seemlessly into a Matlab implementation. We compared the speed of our Fourierbased implementation in Matlab against the classical wavelet transform implementation which is available in the latest Matlab Wavelet Toolbox [71]. For this purpose, the image size is taken $M \times M$ where $M$ varies from 128 to 1024 in steps of 16 . This step size ensures a decomposition depth of 16 quincunx iterations or eight separable iterations. In Fig. 10, we show timings obtained on an $2 \mathrm{GHz}$ PowerPC processor (Apple G5). The fluctuations of the Fourier-based method are due to the specific implementation of the FFT [72] as used by Matlab. However, the general trend shows that the Fourier-based implementation appears to be competitive for image sizes up to about $512 \times 512$.

Finally, we note that the current algorithm can be somewhat further improved at the cost of a slightly more complicated implementation. In particular, the analysis phase can be made as fast as the synthesis one by reducing the size of the high-pass inverse FFT at odd iterations [22].

\section{CONCLUSION}

In this paper, we have proposed to use isotropic polyharmonic B-splines to build a new family of wavelet bases. These B-splines are nonseparable basis functions that are localized versions of generalized thin plate splines, and that converge toward a Gaussian as the order increases due to an improved localization operator that we have introduced. In the second part of the paper, we focused on the 2-D quincunx subsampling scheme to construct wavelet decompositions. Based on the function design in the continuous domain, we derived the suitable scaling and wavelet filters that we need for a fast discrete wavelet transform. Three flavors of semi-orthogonal designs were presented: orthonormal, B-spline, and dual. The B-spline-type wavelets converge to a sum of four Gabor atoms as the order increases. We also highlighted a fast implementation using FFTs.

\section{APPENDIX I \\ UPPER RIESZ BOUND}

We want to evaluate an upper bound over $\omega \in \mathbb{R}^{N}$ for the the autocorrelation (18). First, we can restrict the values of $\boldsymbol{\omega}$ to $[-\pi, \pi]^{N}$ since the autocorrelation is $2 \pi$-periodic in every component $\omega_{k}$ of $\boldsymbol{\omega}$. Second, we easily check from (10) that $\hat{\beta}_{\gamma}(\boldsymbol{\omega}) \leq 1$ which means that $V_{\gamma}\left(e^{j \omega}\right) \leq\|\boldsymbol{\omega}\|^{\gamma} \leq(\sqrt{N} \pi)^{\gamma}$.

Moreover, when $\left|\omega_{k}\right| \leq \pi$, the inequality $\left|\omega_{k}-2 \pi n_{k}\right| \geq$ $\pi\left|n_{k}\right|$ is valid for all $n_{k} \in \mathbb{Z}$. This implies that

$$
\|\boldsymbol{\omega}-2 \pi \mathbf{n}\| \geq \pi\|\mathbf{n}\|
$$




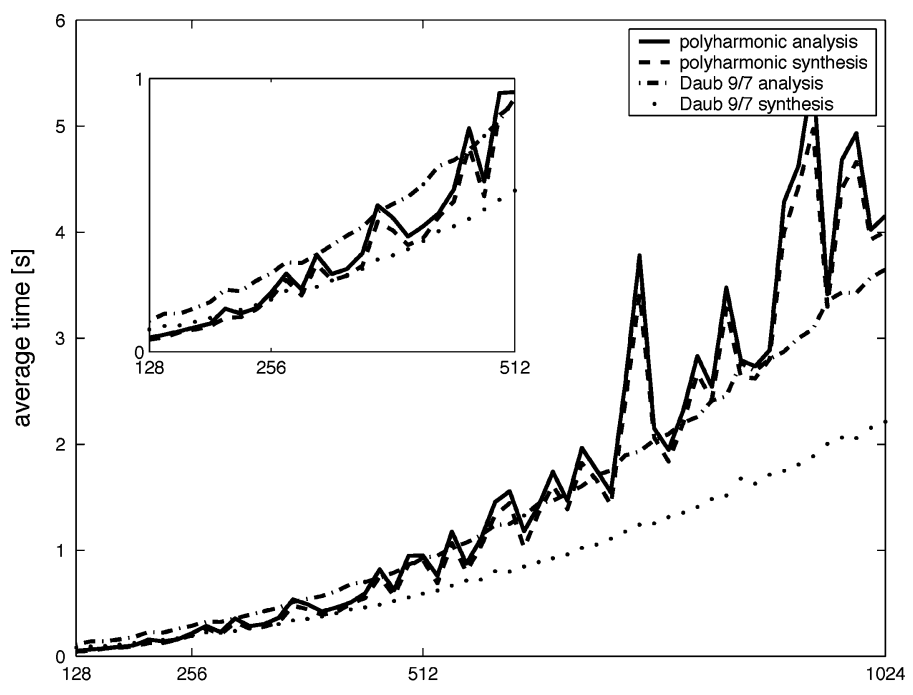

Fig. 10. Execution times in seconds corresponding to the Fourier-based wavelet transform algorithm (blue lines) for the polyharmonic B-spline wavelet transform and Matlab's Wavelet Toolbox (red lines) for the Daubechies 9/7 (JPEG2000) wavelet transform. The size of the test image is $M \times M$ and varies from 128 to 1024 with a step of 16 . The fluctuations of the Fourier-based algorithm are due to the specific implementation of the FFT algorithm.

and, finally

$$
\begin{aligned}
A_{\gamma}\left(e^{j \boldsymbol{\omega}}\right) & =\left|\hat{\beta}_{\gamma}(\boldsymbol{\omega})\right|^{2}+\sum_{\mathbf{n} \in \mathbb{Z}^{N} \backslash\{\mathbf{0}\}} \frac{V_{2 \gamma}\left(e^{j \boldsymbol{\omega}}\right)}{\|\boldsymbol{\omega}+2 \mathbf{n} \pi\|^{2 \gamma}} \\
& \leq 1+\sum_{\mathbf{n} \in \mathbb{Z}^{N} \backslash\{\mathbf{0}\}} \frac{(\sqrt{N} \pi)^{2 \gamma}}{\|\boldsymbol{\omega}+2 \mathbf{n} \pi\|^{2 \gamma}} \\
& \leq 1+N^{\gamma} \sum_{\mathbf{n} \in \mathbb{Z}^{N} \backslash\{\mathbf{0}\}} \frac{1}{\|\mathbf{n}\|^{2 \gamma}}
\end{aligned}
$$

which is known to be bounded whenever $2 \gamma>N$. Note that this bound is not sharp as it tends to increase when $\gamma$ increases.

\section{APPENDIX II \\ CONVERGENCE OF THE ISOTROPIC POLYHARMONIC B-SPLINE WAVELET TO GABOR WAVELET}

In this Appendix, we derive the asymptotic form of the bidimensional (i.e., $N=2$ ) isotropic polyharmonic B-spline wavelet. Before showing that it converges to the sum of four Gabor atoms, symmetrically placed in the frequency domain, we introduce the following lemma.

Lemma 1: For $\gamma \geq 3$, the autocorrelation filter $A_{\gamma}\left(e^{j \boldsymbol{\omega}}\right)$ of the bidimensional isotropic polyharmonic B-spline is bounded over $\mathbb{R}^{2}$, as follows:

$$
\begin{aligned}
\sum_{\|\mathbf{n}\|^{2} \leq 2}\left|\hat{\beta}_{\gamma}([\boldsymbol{\omega}]+2 \pi \mathbf{n})\right|^{2} & \leq A_{\gamma}\left(e^{j \boldsymbol{\omega}}\right) \\
\leq & (1+\epsilon) \sum_{\|\mathbf{n}\|^{2} \leq 2}\left|\hat{\beta}_{\gamma}([\boldsymbol{\omega}]+2 \pi \mathbf{n})\right|^{2}
\end{aligned}
$$

where $\epsilon=12 \sqrt{3}(2 / 3)^{\gamma}$ tends to zero exponentially fast as $\gamma \rightarrow$ $\infty$.

The notation $[\omega]$ stands for the unique 2-D vector in $\left[-\pi, \pi\left[^{2}\right.\right.$ such that $\boldsymbol{\omega}-[\boldsymbol{\omega}]=2 \mathbf{n} \pi$ for some 2-D integer $\mathbf{n}$.

Proof: We first observe that we can restrict the range of values of $\omega$ to $[-\pi, \pi]^{2}$ because the autocorrelation filter is $2 \pi$-periodic. Since the lower bound is trivial we concentrate on the upper bound. We use (53) over the autocorrelation sum restricted to $\|\mathbf{n}\|^{2} \geq 3$, and find

$$
\begin{aligned}
A_{\gamma}\left(e^{j \omega}\right) & \leq \sum_{\|\mathbf{n}\|^{2} \leq 2}\left|\hat{\beta}_{\gamma}(\boldsymbol{\omega}+2 \pi \mathbf{n})\right|^{2}+\sum_{\|\mathbf{n}\|^{2} \geq 3} \frac{V_{2 \gamma}\left(e^{j \boldsymbol{\omega}}\right)}{\|\pi \mathbf{n}\|^{2 \gamma}} \\
& \leq \sum_{\|\mathbf{n}\|^{2} \leq 2}\left|\hat{\beta}_{\gamma}(\boldsymbol{\omega}+2 \pi \mathbf{n})\right|^{2}+\left|\hat{\beta}_{\gamma}(\boldsymbol{\omega})\right|^{2} \sum_{\|\mathbf{n}\|^{2} \geq 3} \frac{\|\boldsymbol{\omega}\|^{2 \gamma}}{\|\pi \mathbf{n}\|^{2 \gamma}} \\
& \leq \sum_{\|\mathbf{n}\|^{2} \leq 2}\left|\hat{\beta}_{\gamma}(\boldsymbol{\omega}+2 \pi \mathbf{n})\right|^{2}\left(1+\sum_{\|\mathbf{n}\|^{2} \geq 3} \frac{\|\boldsymbol{\omega}\|^{2 \gamma}}{\|\pi \mathbf{n}\|^{2 \gamma}}\right) \\
& \leq \sum_{\|\mathbf{n}\|^{2} \leq 2}\left|\hat{\beta}_{\gamma}(\boldsymbol{\omega}+2 \pi \mathbf{n})\right|^{2}\left(1+\sum_{\|\mathbf{n}\|^{2} \geq 3} \frac{2^{\gamma}}{\|\mathbf{n}\|^{2 \gamma}}\right) .
\end{aligned}
$$

We, thus, have to evaluate the quantity $2^{\gamma} \sum_{\|\mathbf{n}\|^{2}>3}\|\mathbf{n}\|^{-2 \gamma}$. We denote by $\nu_{N}$ the number of (signed) integer solutions to the Diophantine equation: $n_{1}^{2}+n_{2}^{2}=N$. Obviously, $\nu_{N} \leq 2 \times 2 \sqrt{N}$ because $n_{1}$ is necessarily in $[-\sqrt{N}, \sqrt{N}]$ and $n_{2}$ is uniquely determined by $n_{1}$ up to a sign. Then, we find

$$
\begin{aligned}
2^{\gamma} \sum_{\|\mathbf{n}\|^{2} \geq 3}\|\mathbf{n}\|^{-2 \gamma} & =2^{\gamma} \sum_{N \geq 3} \frac{\nu_{N}}{N^{\gamma}} \\
& \leq 2^{\gamma+2} \sum_{N \geq 3} \frac{1}{N^{\gamma-1 / 2}}
\end{aligned}
$$

which is convergent because $\gamma-1 / 2>1$. Now, it is well known (see [73]) that the remainder of the Riemann zeta function is bounded according to $\sum_{N>N_{0}} N^{-s} \leq N_{0}^{-s+1} /(s-1)$. Applying this formula with $N_{0}=4$ and $s=\gamma-1 / 2$, and taking into account that $\gamma \geq 3$, we find

$$
\begin{array}{r}
2^{\gamma} \sum_{\|\mathbf{n}\|^{2} \geq 3}\|\mathbf{n}\|^{-2 \gamma} \leq 2^{\gamma+2}\left(\frac{1}{3^{\gamma-1 / 2}}+\frac{1}{(\gamma-3 / 2) 3^{\gamma-3 / 2}}\right) \\
\leq 12 \sqrt{3}\left(\frac{2}{3}\right)^{\gamma}
\end{array}
$$

which proves the lemma. 
Armed with this lemma, we can now proceed to the proof of our convergence theorem. The isotropic polyharmonic B-spline wavelet has been defined as

$$
2 \hat{\psi}\left(\mathbf{D}^{\mathrm{T}} \boldsymbol{\omega}\right)=-e^{-j \omega_{1}} B_{\gamma}\left(e^{j(\boldsymbol{\omega}+\boldsymbol{\pi})}\right) A_{\gamma}\left(e^{j(\boldsymbol{\omega}+\boldsymbol{\pi})}\right) \hat{\beta}_{\gamma}(\boldsymbol{\omega})
$$

Thanks to Lemma 1, the autocorrelation filter can be replaced by a finite sum when $\gamma \rightarrow \infty$, i.e.,

$$
\begin{aligned}
2 \hat{\psi}\left(\mathbf{D}^{\mathrm{T}} \boldsymbol{\omega}\right) & \\
= & -e^{-j \omega_{1}} \\
& \times \sum_{\|\mathbf{n}\|^{2} \leq 2}(\underbrace{\hat{\beta}_{2}([\boldsymbol{\omega}+\boldsymbol{\pi}]+2 \pi \mathbf{n}) B_{1}\left(e^{j(\boldsymbol{\omega}+\boldsymbol{\pi})}\right) \hat{\beta}_{1}(\boldsymbol{\omega})}_{r_{\mathbf{n}}(\boldsymbol{\omega})})^{\gamma} \\
& \times\left(1+\mathcal{O}\left((2 / 3)^{\gamma}\right)\right) .
\end{aligned}
$$

By inspection, we observe that

$\sup r_{\mathbf{n}}(\boldsymbol{\omega})$

$\boldsymbol{\omega} \in \mathbb{R}^{2}$

$$
\approx \begin{cases}0.05, & \text { for } \mathbf{n}=(-1,-1),(-1,1),(1,-1),(1,1) \\ 0.16, & \text { for } \mathbf{n}=(-1,0),(0,-1),(0,1),(1,0) \\ 0.4, & \text { for } \mathbf{n}=(0,0)\end{cases}
$$

which shows that, as $\gamma \rightarrow \infty, r_{\mathbf{0}}(\omega)^{\gamma}$ dominates the other $r_{\mathbf{n}}(\boldsymbol{\omega})^{\gamma}$ terms, i.e.,

$$
\hat{\psi}(\boldsymbol{\omega}) \approx-\frac{1}{2} e^{-j \frac{\omega_{1}+\omega_{2}}{2}} r_{\mathbf{0}}\left(\mathbf{D}^{-\mathrm{T}} \boldsymbol{\omega}\right)^{\gamma}
$$

As one can readily observe, $r_{\mathbf{0}}(\boldsymbol{\omega})$ has a fourfold symmetry: $r_{0}\left(\omega_{1}, \omega_{2}\right)=r_{0}\left(-\omega_{1}, \omega_{2}\right)=r_{0}\left(\omega_{1},-\omega_{2}\right)=r_{\mathbf{0}}\left(-\omega_{1},-\omega_{2}\right)$. By inspection again, $r_{0}(\omega)$ reaches its maximum $M \approx 0.4$ at $\boldsymbol{\omega}=\left(\omega^{\prime}, \omega^{\prime}\right)$, and at its other three symmetric positions $\left(-\omega^{\prime}, \omega^{\prime}\right),\left(\omega^{\prime},-\omega^{\prime}\right)$, and $\left(-\omega^{\prime},-\omega^{\prime}\right)$, where $\omega^{\prime} \approx 5.074$. This means that $\hat{\psi}(\boldsymbol{\omega})$ reaches its maximum at $\boldsymbol{\omega}=\left(\omega^{\prime}, 0\right),\left(-\omega^{\prime}, 0\right),\left(0, \omega^{\prime}\right)$ and $\left(0,-\omega^{\prime}\right)$.

In the neighborhood of $\omega^{\prime}=\left(\omega^{\prime}, 0\right)$, we consider the Taylor development

$$
\begin{aligned}
\ln r_{(0,0)}\left(\mathbf{D}^{-\mathrm{T}} \boldsymbol{\omega}\right)=\ln M & -\frac{1}{2}\left(\frac{\omega_{1}-\omega^{\prime}}{s_{1}}\right)^{2} \\
& -\frac{1}{2}\left(\frac{\omega_{2}}{s_{2}}\right)^{2}+\mathcal{O}\left(\left\|\boldsymbol{\omega}-\boldsymbol{\omega}^{\prime}\right\|^{3}\right)
\end{aligned}
$$

with

$$
s_{1} \approx 2.7, \quad s_{2} \approx 2.36
$$

Using the central limit theorem, we obtain

$$
\begin{aligned}
\hat{\psi}(\boldsymbol{\omega}) \approx & -\frac{M^{\gamma}}{2} e^{-j \frac{\omega_{1}+\omega_{2}}{2}}\left(\hat{g}\left(\omega_{1}, \omega_{2}\right)+\hat{g}\left(-\omega_{1}, \omega_{2}\right)\right. \\
& \left.+\hat{g}\left(\omega_{2}, \omega_{1}\right)+\hat{g}\left(\omega_{2},-\omega_{1}\right)\right)
\end{aligned}
$$

with

$$
\begin{aligned}
& \hat{g}(\boldsymbol{\omega})=e^{-\frac{\gamma}{2}\left(\frac{\omega_{1}-\omega^{\prime}}{s_{1}}\right)^{2}-\frac{\gamma}{2}\left(\frac{\omega_{2}}{s_{2}}\right)^{2}} \\
& \longleftrightarrow \quad g(\mathbf{x}) \frac{s_{1} s_{2}}{2 \pi \gamma} e^{j \omega^{\prime} x_{1}} e^{-\frac{s_{1}^{2} x_{1}^{2}+s_{2}^{2} x_{2}^{2}}{2 \gamma}} .
\end{aligned}
$$

Finally, we state that the inverse Fourier transform of both sides is equivalent for large $\gamma$, providing

$$
\begin{aligned}
\psi(\mathbf{x}) \approx & -\frac{M^{\gamma} s_{1} s_{2}}{2 \pi \gamma} \\
& \times\left(\cos \left(\omega^{\prime} x_{1}-\frac{\omega^{\prime}}{2}\right) e^{-\frac{s_{1}^{2}\left(x_{1}-\frac{1}{2}\right)^{2}+s_{2}^{2}\left(x_{2}-\frac{1}{2}\right)^{2}}{2 \gamma}}\right. \\
& \left.+\cos \left(\omega^{\prime} x_{2}-\frac{\omega^{\prime}}{2}\right) e^{-\frac{s_{1}^{2}\left(x_{2}-\frac{1}{2}\right)^{2}+s_{2}^{2}\left(x_{1}-\frac{1}{2}\right)^{2}}{2 \gamma}}\right) .
\end{aligned}
$$

\section{APPENDIX III}

\section{CONVERGENCE OF THE ORTHONORMAL POLYHARMONIC B-SPLINE TO THE SINC FUNCTION}

We want to show that $\hat{\beta}_{\gamma}^{\perp}(\boldsymbol{\omega})$ tends to the function $\operatorname{rect}\left(\left(\omega_{1}\right) /(2 \pi)\right) \cdot \operatorname{rect}\left(\left(\omega_{2}\right) /(2 \pi)\right)$ indicator of the square $[-\pi, \pi]^{2}$. Assume that $\boldsymbol{\omega}$ belongs to $[-\pi(1-\eta), \pi(1-\eta)]^{2}$ where $\eta$ is some small positive quantity. Then, according to the definition (20) and Lemma 1, we have

$$
\begin{aligned}
1 \geq\left|\hat{\beta}_{\gamma}^{\perp}(\boldsymbol{\omega})\right|^{2} & \geq \frac{\left|\hat{\beta}_{\gamma}(\boldsymbol{\omega})\right|^{2}}{\sum_{\|\mathbf{n}\|^{2} \leq 2}\left|\hat{\beta}_{\gamma}(\boldsymbol{\omega}+2 \pi \mathbf{n})\right|^{2}}+\mathcal{O}(2 / 3)^{\gamma} \\
& \geq \frac{1}{1+\sum_{\|\mathbf{n}\|=1}^{\sqrt{2}} \frac{|\boldsymbol{\omega}|^{2 \gamma}}{|\boldsymbol{\omega}+2 \pi \mathbf{n}|^{2 \gamma}}}+\mathcal{O}(2 / 3)^{\gamma} .
\end{aligned}
$$

Moreover, using the obvious inequality $\left|n_{1}\right|+\left|n_{2}\right| \leq\|\mathbf{n}\|^{2}$ and the hypothesis that $\left|\omega_{k}\right| \leq \pi(1-\eta)$, we find

$$
\begin{aligned}
\|\boldsymbol{\omega}+2 \mathbf{n} \pi\|^{2}= & \|\boldsymbol{\omega}\|^{2}+4 \pi \mathbf{n}^{\mathrm{T}} \boldsymbol{\omega}+4 \pi^{2}\|\mathbf{n}\|^{2} \\
\geq & \|\boldsymbol{\omega}\|^{2}-4 \pi^{2}(1-\eta)\left(\left|n_{1}\right|+\left|n_{2}\right|\right) \\
& +4 \pi^{2}\|\mathbf{n}\|^{2} \\
\geq & \|\boldsymbol{\omega}\|^{2}+4 \pi^{2} \eta\|\mathbf{n}\|^{2} \\
\geq & \|\boldsymbol{\omega}\|^{2}+4 \pi^{2} \eta\|\mathbf{n}\|^{2} \cdot \frac{\|\boldsymbol{\omega}\|^{2}}{2 \pi^{2}(1-\eta)^{2}} \\
\geq & \frac{1+\eta^{2}}{(1-\eta)^{2}}\|\boldsymbol{\omega}\|^{2} \quad \text { whenever }\|\mathbf{n}\| \geq 1
\end{aligned}
$$

This means that $\boldsymbol{\omega} \in[-\pi(1-\eta), \pi(1-\eta)]^{2}$

$$
1 \geq\left|\hat{\beta}_{\gamma}^{\perp}(\boldsymbol{\omega})\right|^{2} \geq \underbrace{\frac{1}{1+\mathcal{O}\left(\left(\frac{(1-\eta)^{2}}{1+\eta^{2}}\right)^{\gamma}\right)}+\mathcal{O}(2 / 3)^{\gamma}}_{\longrightarrow \gamma \rightarrow \infty} .
$$

Since this is true for all $\eta>0$, we have

$$
\left.\left|\hat{\beta}_{\gamma}^{\perp}(\boldsymbol{\omega})\right|_{\gamma \rightarrow \infty}^{2} 1 \text { for all } \boldsymbol{\omega} \in\right]-\pi, \pi\left[{ }^{2} .\right.
$$

Thanks to the orthonormality relation

$$
1=\sum_{\mathbf{n} \in \mathbb{Z}^{2}}\left|\hat{\beta}_{\gamma}^{\perp}(\boldsymbol{\omega}+2 \mathbf{n} \pi)\right|^{2}
$$

we also find that $\left|\hat{\beta}_{\gamma}^{\perp}(\boldsymbol{\omega})\right|^{2} \rightarrow 0$ in every square

$$
\boldsymbol{\omega} \in 2 \mathbf{n} \pi+]-\pi, \pi\left[^{2} \text { with } \mathbf{n} \in \mathbb{Z} \backslash\{\mathbf{0}\} .\right.
$$

Finally, because of the inequality $\left|\hat{\beta}_{\gamma}([\boldsymbol{\omega}])\right|^{2} \leq A_{\gamma}\left(e^{j \omega}\right)$ (see Lemma 1) we also have that $\left|\hat{\beta}_{\gamma}^{\perp}(\boldsymbol{\omega})\right|^{2} \leq\|[\boldsymbol{\omega}]\|^{2 \gamma} /\|\boldsymbol{\omega}\|^{2 \gamma}$ which implies that $\left|\hat{\beta}_{\gamma}^{\perp}(\mathbf{n} \pi)\right|^{2} \leq\left(2 \pi^{2}\right)^{\gamma} /\|\mathbf{n} \pi\|^{2 \gamma} \leq(2 / 3)^{\gamma}$ when $\|\mathbf{n}\|^{2} \geq 3$. Since this expression tends to 0 as $\gamma \rightarrow \infty$, we can now conclude that $\left|\hat{\beta}_{\gamma}^{\perp}(\boldsymbol{\omega})\right|^{2}$ tends to zero when 
(a)

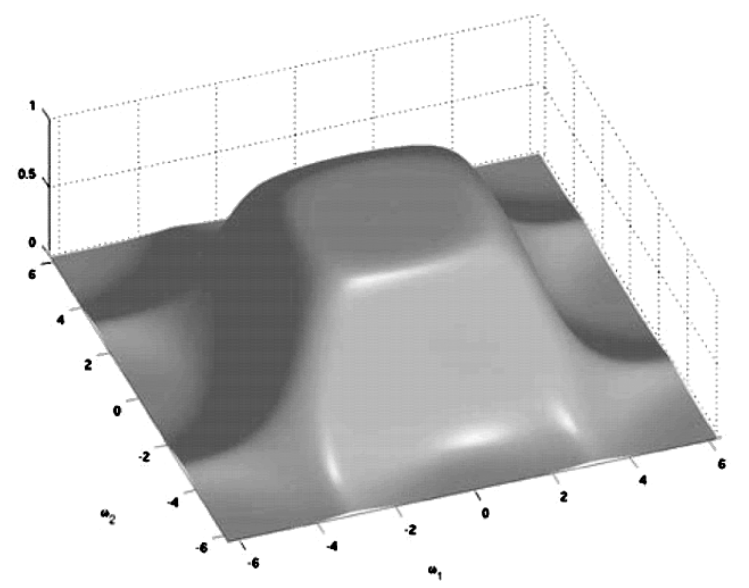

(b)

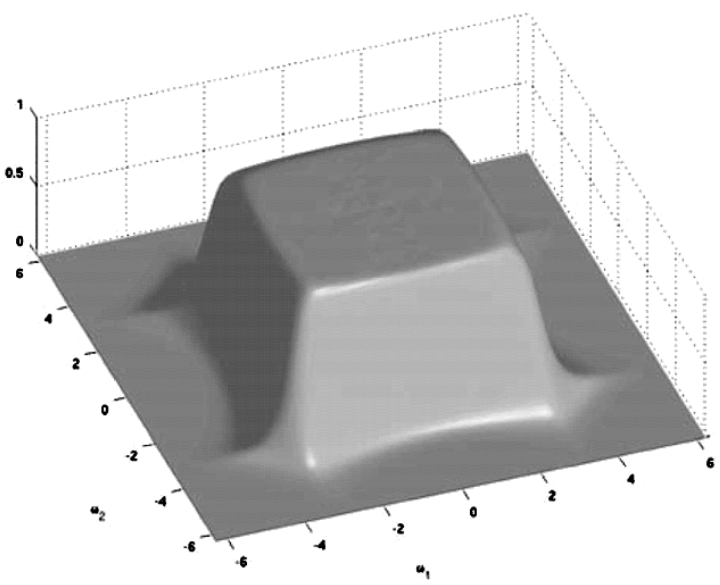

Fig. 11. Frequency responses $\left|\hat{\beta}_{\gamma}^{\perp}\right|$ for (a) $\gamma=4$ and (b) $\gamma=10$.

$\boldsymbol{\omega} \in \mathbb{R}^{2} \backslash[-\pi, \pi]^{2}$, which proves the convergence of $\hat{\beta}_{\gamma}^{\perp}(\boldsymbol{\omega})$ to $\operatorname{rect}\left(\left(\omega_{1}\right) /(2 \pi)\right) \cdot \operatorname{rect}\left(\left(\omega_{2}\right) /(2 \pi)\right)$. Fig. 11 illustrates the convergence.

\section{ACKNOWLEDGMENT}

The authors would like to thank the anonymous reviewers for their valuable remarks and suggestions.

\section{REFERENCES}

[1] S. Mallat, "A theory for multiresolution signal decomposition: The wavelet decomposition," IEEE Trans. Pattern Anal. Mach. Intell., vol. 11, no. 5, pp. 674-693, May 1989.

[2] I. Daubechies, Ten Lectures on Wavelets. Philadelphia, PA: SIAM, 1992.

[3] M. Unser and T. Blu, "Wavelet theory demystified," IEEE Trans. Signal Process., vol. 51, no. 2, pp. 470-483, Feb. 2003.

[4] E. J. Candès and D. L. Donoho, "Curvelets-A surprisingly effective nonadaptive representation for objects with edges," in Curve and Surface Fitting. Nashville, TN: Vanderbilt Univ. Press, 1999.

[5] — , "Ridgelets: A key to higher-dimensional intermittency?," Phil. Trans. R. Soc. London A, pp. 2495-2509, 1999.

[6] J. L. Starck, E. J. Candès, and D. L. Donoho, "The curvelet transform for image denoising," IEEE Trans. Image Process., vol. 11, no. 6, pp. 670-684, Jun. 2002.

[7] V. Velisavljevic, P. L. Dragotti, and M. Vetterli, "Directional wavelet transforms and frames," in Proc. IEEE Int. Conf. Image Processing, vol. 3, 2002, pp. 589-592.

[8] P. Vandergheynst and J.-F. Gobbers, "Directional dyadic wavelet transforms: Design and algorithms," IEEE Trans. Image Process., vol. 11, no. 4, pp. 363-372, Apr. 2002.

[9] M. N. Do and M. Vetterli, "The finite ridgelet transform for image representation," IEEE Trans. Image Process., vol. 12, no. 1, pp. 16-28, Jan. 2003.

[10] - Beyond Wavelets. New York: Academic, 2003. Contourlets.

[11] V. Velisavljevic, B. Beferull-Lozano, M. Vetterli, and P. L. Dragotti, "Discrete multi-directional wavelet bases," in Proc. IEEE Int. Conf. Image Processing, vol. 1, 2003, pp. 1025-1028.

[12] M. Unser and T. Blu, "Fractional wavelets, derivatives, and Besov spaces," presented at the SPIE Conf. Mathematical Imaging: Wavelet Applications in Signal and Image Processing X, San Diego, CA, 2003.

[13] T. Blu and M. Unser, "On the fractional approximation order and Sobolev regularity of scaling functions," SIAM J. Math. Anal., to be published.

[14] E. P. Simoncelli and E. H. Adelson, "Non-separable extensions of quadrature mirror filters to multiple dimensions," in Proc. IEEE, vol. 78, Jun. 1990, pp. 652-664.

[15] G. Karlsson and M. Vetterli, "Theory of two-dimensional multirate filter banks," IEEE Trans. Acoust., Speech, Signal Process., vol. 38, no. 6, pp. 925-937, Dec. 1990.

[16] E. Viscito and J. P. Allebach, "The analysis and design of multidimensional FIR perfect reconstruction filter banks for arbitrary sampling lattices," IEEE Trans. Circuits Syst., vol. 38, no. 1, pp. 29-42, Jan. 1991.
[17] J. Kovačević and M. Vetterli, "Nonseparable multidimensional perfect reconstruction filter banks and wavelet bases for $\mathbb{R}^{n}$," IEEE Trans. Inf. Theory, vol. 38, no. 2, pp. 533-555, Apr. 1992.

[18] - "Nonseparable two- and three-dimensional wavelets," IEEE Trans. Signal Process., vol. 43, no. 5, pp. 1269-1273, May 1995.

[19] B. Han and R.-Q. Jia, "Quincunx fundamental refinable functions and quincunx biorthogonal wavelets," Math. Comput., vol. 71, no. 237, pp. 165-196, 2002.

[20] J. H. McClellan, "The design of two-dimensional digital filters by transformations," in Proc. 7th Annu. Princeton Conf. Information Sciences and Systems, Princeton, NJ, 1973, pp. 247-251.

[21] D. E. Dudgeon and R. M. Mersereau, Multidimensional Digital Signal Processing. Englewood Cliffs, NJ: Prentice-Hall, 1984.

[22] M. Feilner, D. Van De Ville, and M. Unser, "An orthogonal family of quincunx wavelets with continuously-adjustable order," IEEE Trans. Image Process., vol. 14, no. 4, pp. 449-510, Apr. 2005.

[23] T. Blu and M. Unser, "Wavelets, fractals, and radial basis functions," IEEE Trans. Signal Process., vol. 50, no. 3, pp. 543-553, Mar. 2002.

[24] I. J. Schoenberg, Cardinal Spline Interpolation. Philadelphia, PA: SIAM, 1973, vol. 12.

[25] R. L. Harder and R. N. Demarais, "Interpolation using surface splines," J. Aircraft, vol. 9, pp. 189-191, 1972.

[26] J. Duchon, "Interpolation des fonctions de deux variables suivant le principe de la flexion des plaques minces," RAIRO Anal. Numer, vol. 10, no. 12 , pp. 5-12, 1976.

[27] - Constructive Theory of Functions of Several Variables. Berlin, Germany: Springer, 1977, pp. 85-100. Splines minimizing rotation-invariant semi-norms in Sobolev spaces.

[28] R. Franke, "A critical comparison of some methods for interpolation of scattered data," Math. Comput., vol. 38, pp. 181-200, 1982.

[29] N. Dyn and D. Levin, "Iterative solution of systems originating from integral equations and surface interpolation," Numer. Anal., vol. 20, no. 2, pp. 377-390, 1983.

[30] M. D. Buhmann, "Radial basis functions," Acta Numer., pp. 1-38, 2000.

[31] M. Fenn and G. Steidl, "Fast NFFT based summation of radial functions," Sampl. Theory Signal Image Process., vol. 3, no. 1, pp. 1-28, 2004.

[32] W. R. Madych and S. A. Nelson, "Polyharmonic cardinal spline," J. Approx. Theory, vol. 60, pp. 141-156, 1990.

[33] - "Polyharmonic cardinal splines: A minimization property," J. Approx. Theory, vol. 63, pp. 303-320, 1990.

[34] O. Kounchev, Multivariate polysplines: Applications to Numerical and Wavelet Analysis. New York: Academic, 2001.

[35] C. Rabut, "Elementary $m$-harmonic cardinal B-splines," Numer. Algorithms, vol. 2, pp. 39-62, 1992.

[36] C. Rabut, "High level $m$-harmonic cardinal B-splines," Numer. Algorithms, vol. 2, pp. 63-84, 1992.

[37] M. L. Glasser and I. J. Zucker, "chapter lattice sums in theoretical chemistry," in Theoretical Chemistry: Advances and Perspectives. New York: Academic, 1980, vol. 5, pp. 69-139.

[38] D. Shanks, "Calculation and applications of Epstein Zeta functions," Math. Comput., vol. 29, no. 129, pp. 271-287, 1975.

[39] E. Elizalde, "Zeta functions: Formulas and applications," J. Comput. Appl. Math., vol. 118, pp. 125-142, 2000.

[40] C. de Boor, R. Devore, and A. Ron, "Approximation from shift invariant subspaces of $L^{2}\left(\mathbb{R}^{d}\right)$," Trans. Amer. Math. Soc., vol. 341, pp. 787-806, 1994. 
[41] T. Blu and M. Unser, "Approximation error for quasiinterpolators and multi-wavelet expansions," Appl. Comput. Harmon. Anal., vol. 6, no. 2, pp. 219-251, 1999.

[42] W. R. Madych, "Polyharmonic splines, multiscale analysis, and entire functions," Int. Ser. Numer. Math., vol. 94, pp. 205-216, 1990.

[43] - " "Some elementary properties of multiresolution analyzes of $L_{2}\left(\mathbb{R}^{n}\right)$," in Wavelets: A Tutorial in Theory and Applications. Boston, MA: Academic, 1992, pp. 259-294.

[44] C. Micchelli, C. Rabut, and F. I. Utreras, "Using the refinement equation for the construction of pre-wavelets III: Elliptic splines," Numer. Algorithms 1, vol. 4, pp. 331-352, 1991.

[45] A. Cohen and I. Daubechies, "Non-separable bidimensional wavelet bases," Revista Matematica Iberoamer, vol. 9, pp. 51-137, 1993.

[46] M. Vetterli, "Multi-dimensional sub-band coding: Some theory and algorithms," Signal Process., vol. 6, no. 2, pp. 97-112, 1984.

[47] J.-C. Feauveau, "Analyse multirésolution par ondelettes non orthogonales et bancs de filtres numériques," Ph.D. dissertation, Univ. Paris Sud, Paris, France, 1990

[48] F. Nicolier, O. Laligant, and F. Truchetet, "B-spline quincunx wavelet transform and implementation in Fourier domain," Proc. SPIE, vol. 3522, pp. 223-234, Nov. 1998.

[49] — , "Discrete wavelet transform implementation in fourier domain for multidimensional signal," J. Electron. Imag., vol. 11, no. 3, pp. 338-346, 2002

[50] M. Unser, A. Aldroubi, and M. Eden, "A family of polynomial spline wavelet transforms," Signal Process., vol. 30, pp. 141-162, 1993.

[51] D. Gabor, "Theory of communication," J. Inst. Elect. Eng., vol. 93, no. III, pp. 429-457, 1946

[52] M. J. Bastiaans, "Gabor's expansion of a signal into gaussian elementary signals," Proc. IEEE, vol. 68, no. 5, pp. 538-539, May 1980

[53] M. Porat and Y. Y. Zeevi, "The generalized Gabor scheme of image representation in biological and machine vision," IEEE Trans. Pattern Anal. Mach. Intell., vol. 10, no. 4, pp. 452-468, Apr. 1988.

[54] I. Daubechies, "The wavelet transform, time-frequency localization, and signal analysis," IEEE Trans. Inf. Theory, vol. 36, no. 5, pp. 961-1005, Oct. 1990.

[55] J. Wexler and S. Raz, "Discrete Gabor expansion," Signal Process., vol. 21, pp. 207-220, 1990

[56] T. S. Lee, "Image representation using 2-D Gabor wavelets," IEEE Trans. Pattern Anal. Mach. Intell., vol. 18, no. 10, pp. 959-971, Oct. 1996.

[57] H. G. Feichtinger and T. Strohmer, Eds., Advances in Gabor Analysis. Berlin, Germany: Applied and Numerical Harmonic Analysis, 2002

[58] J. F. Canny, "A computational approach to edge detection," IEEE Trans. Pattern Anal. Mach. Intell., vol. PAMI-8, no. 6, pp. 679-698, Jun. 1986.

[59] M. Basu, "Gaussian-based edge-detection methods-a survey," IEEE Trans. Syst., Man, Cybern. C, Appl. Rev., vol. 32, no. 3, pp. 252-260, Aug. 2002.

[60] M. Turner, "Texture discrimitation by Gabor functions," Biol. Cybern., vol. 55, pp. 71-82, 1986.

[61] J. G. Daugman, "Uncertainty relation for resolution in space, spatial frequency, and orientation optimized by two-dimensional visual cortical filters," J. Opt. Soc. Amer. A, vol. 2, no. 7, pp. 1160-1169, 1985.

[62] D. J. Field, "Relations between the statistics of natural images and the response properties of cortical cells," J. Opt. Soc. Amer. A, vol. 4, no. 12, pp. 2379-2394, 1987.

[63] B. A. Wandell, Foundations of Vision. Sunderland, MA: Sinauer Associates, 1995.

[64] D. Van De Ville, T. Blu, and M. Unser, "Wavelets versus resels in the context of fMRI: Establishing the link with SPM," in Proc. SPIE Symp. Optical Science and Technology: Wavelets X, vol. 5207, San Diego, CA, 2003, pp. 417-425.

[65] H. J. Park and H. S. Yang, "Invariant object detection based on evidence accumulation and Gabor features," Pattern Recognit. Lett., vol. 22, pp. 869-882, 2001.

[66] V. Krüger and G. Sommer, "Gabor wavelet networks for efficient head pose estimation," Image Vis. Comput., vol. 20, no. 9-10, pp. 665-672, 2002.

[67] V. Kyrki, J.-K. Kamarainen, and H. Kälviäinen, "Simple gabor feature space for invariant object recognition," Pattern Recognit. Lett., vol. 25, pp. 311-318, 2004

[68] T. Kanade and F. Kagalwala, "Reconstructing specimens using DIC microscope images," IEEE Trans. Syst., Man, Cybern. B: Cybern., vol. 33, no. 5 , pp. 728-737, Oct. 2003

[69] B. Bacchelli, M. Bozzini, and C. Rabut, "A fast wavelet algorithm for multi-dimensional signal using polyharmonic splines," in Curve and Surface Fitting. Brentwood, TN: NashboroPress, 2003, pp. 21-30.
[70] T. Blu, D. Van De Ville, and M. Unser "Numerical methods for the computation of wavelet correlation sequences," SIAM J. Numer. Anal., to be published.

[71] Wavelet Toolbox 2.2. Natick, MA: The Mathworks, Inc., 2000.

[72] M. Frigo and S. G. Johnson, "FFTW: An adaptive software architecture for the FFT," in Proc. ICASSP, vol. 3, 1998, pp. 1381-1384.

[73] M. Abramowitz and I. A. Stegun, Eds., Handbook of Mathematical Functions with Formulas, Graphs, and Mathematical Table, 10th ed. Washington, DC: Government Printing Office, 1972.

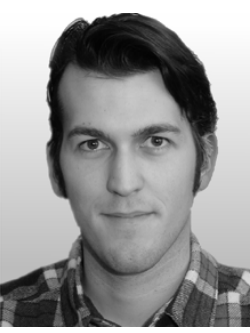

Dimitri Van De Ville (M'02) was born in Dendermonde, Belgium, in 1975. He received the Engineering and $\mathrm{Ph} . \mathrm{D}$. degrees in computer sciences from Ghent University, Ghent, Belgium, in July 1998 and January 2002, respectively.

While studying for the $\mathrm{Ph} . \mathrm{D}$. degree, he was a Research Assistant with the Fund for Scientific Research, Flanders, Belgium, at the Medical Image and Signal Processing Group within the Department of Electronics and Information Systems. He is currently with the Biomedical Imaging Group, Swiss Federal Institute of Technology Lausanne, Lausanne, Switzerland. He is a Technical Editor of the Wavelet Digest, the electronic newsletter of the wavelet community. His current research interests include splines, wavelets, approximation and sampling theory, and biomedical signal and imaging applications, such as fMRI and microscopy imaging.

Dr. Van De Ville is an Associate Editor of the IEEE Signal Processing LETTERS.

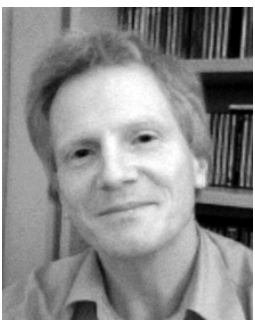

Thierry Blu (M'96) was born in Orléans, France, in 1964. He received the "Diplôme d'ingénieur" from the École Polytechnique, France, in 1986 and from Télécom Paris (ENST), France, in 1988, and the $\mathrm{Ph} . \mathrm{D}$. degree in electrical engineering from ENST in 1996 for a study on iterated rational filterbanks, applied to wideband audio coding.

$\mathrm{He}$ is with the Biomedical Imaging Group, Swiss Federal Institute of Technology, Lausanne, Switzerland, on leave from the France Télécom National Center for Telecommunications Studies (CNET), Issy-les-Moulineaux, France. His research interests include (multi)wavelets, multiresolution analysis, multirate filterbanks, approximation and sampling theory, psychoacoustics, optics, and wave propagation.

Dr. Blu is the recipient of the 2003 best paper award (SP Theory and Methods) from the IEEE Signal Processing Society. He is currently an Associate Editor for the IEEE TRANSACTIONS ON IMAGE PROCESSING.

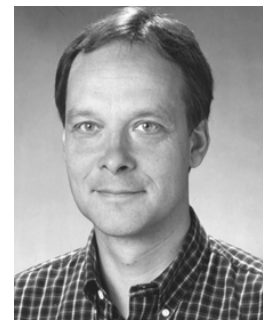

Michael Unser (M'89-SM'94-F'99) received the M.S. (summa cum laude) and Ph.D. degrees in electrical engineering from the Swiss Federal Institute of Technology (EPFL), Lausanne, Switzerland, in 1981 and 1984 , respectively.

From 1985 to 1997 , he was with the Biomedical Engineering and Instrumentation Program, National Institutes of Health, Bethesda, MD, where he headed the Image Processing Group. He is now Professor and Director of the Biomedical Imaging Group, EPFL. $\mathrm{He}$ is the Editor-in-Chief of the Wavelet Digest, the electronic newsletter of the wavelet community. He also served as regular chair for SPIE's Conference on Wavelets from 1993 to 2005. His research area is biomedical image processing. He has a strong interest in sampling theories, multiresolution algorithms, wavelets, and the use of splines for image processing, and he is the author of over 100 published journal papers in these areas.

Dr. Unser is the Associate Editor-in-Chief of the IEEE TRANSACTIONS ON MEDICAL IMAGING. He has acted as Associate Editor or member of the editorial boards for the IEEE SIGNAL PROCESSING MAGAZINE, the IEEE TRANSACTIONS ON IMAGE PROCESSING (1992 to 1995), and the IEEE SignAL Processing LETTERS (1994 to 1998). He was general Co-Chair for the first IEEE International Symposium on Biomedical Imaging (ISBI'2002), Washington, DC, July 7-10, 2002. He received the IEEE Signal Processing Society's 1995 Best Paper Award and the IEEE Signal Processing Society's 2000 Magazine Award. 